\title{
Gas-rich mergers and feedback are ubiquitous amongst starbursting radio galaxies, as revealed by the VLA, IRAM PdBI and Herschel
}

\author{
R. J. Ivison, ${ }^{1,2 \star}$ Ian Smail, ${ }^{3}$ A. Amblard, ${ }^{4}$ V. Arumugam, ${ }^{2}$ C. De Breuck, ${ }^{5}$ \\ B. H. C. Emonts, ${ }^{6}$ I. Feain, ${ }^{6}$ T. R. Greve,${ }^{7}$ M. Haas, ${ }^{8}$ E. Ibar, ${ }^{1}$ M. J. Jarvis, ${ }^{9}$ \\ A. Kovács, ${ }^{10,11}$ M. D. Lehnert, ${ }^{12}$ N. P. H. Nesvadba, ${ }^{13}$ H. J. A. Röttgering, ${ }^{14}$ \\ N. Seymour ${ }^{6}$ and D. Wylezalek ${ }^{5}$ \\ ${ }^{1}$ UK Astronomy Technology Centre, Science and Technology Facilities Council, Royal Observatory, Blackford Hill, Edinburgh EH9 3 HJ \\ ${ }^{2}$ Institute for Astronomy, University of Edinburgh, Blackford Hill, Edinburgh EH9 $3 \mathrm{HJ}$ \\ ${ }^{3}$ Institute for Computational Cosmology, Durham University, South Road, Durham DH1 3 LE \\ ${ }^{4}$ NASA, Ames Research Center, Moffett Field, CA 94035, USA \\ ${ }^{5}$ European Southern Observatory, Karl Schwarzschild Straße 2, 85748 Garching, Germany \\ ${ }^{6}$ CSIRO Astronomy and Space Science, Australia Telescope National Facility, PO Box 76, Epping, NSW 1710, Australia \\ ${ }^{7}$ Department of Physics and Astronomy, University College London, Gower Street, London WC1E $6 B T$ \\ ${ }^{8}$ Astronomisches Institut, Ruhr-Universität Bochum, Universitätsstraße 150, 44801 Bochum, Germany \\ ${ }^{9}$ Centre for Astrophysics Research, Science \& Technology Research Institute, University of Hertfordshire, Hatfield, Herts AL10 9AB \\ ${ }^{10}$ California Institute of Technology, 301-17, 1200 East California Blvd, Pasadena, CA 91125, USA \\ ${ }^{11}$ Institute for Astrophysics, University of Minnesota, 116 Church Street SE, MN 55455, USA \\ ${ }^{12}$ GEPI, Observatoire de Paris, UMR 8111, CNRS, Université Paris Diderot, 5 place Jules Janssen, 92190 Meudon, France \\ ${ }^{13}$ Institut d'Astrophysique Spatiale, Université Paris Sud 11, Orsay, France \\ ${ }^{14}$ Leiden Observatory, University of Leiden, PO Box 9513, 2300 RA Leiden, the Netherlands
}

Accepted 2012 June 17. Received 2012 June 15; in original form 2012 May 10

\begin{abstract}
We report new, sensitive observations of two $z \sim 3-3.5$ far-infrared-luminous radio galaxies, 6C $1909+72$ and B3 J2330+3927, in the ${ }^{12} \mathrm{CO} J=1-0$ transition with the Karl Jansky Very Large Array and at $100-500 \mu \mathrm{m}$ using Herschel, alongside new and archival ${ }^{12} \mathrm{CO} J=4-3$ observations from the Plateau de Bure Interferometer. We introduce a new colour-colour diagnostic plot to constrain the redshifts of several distant, dusty galaxies in our target fields. A bright SMG near 6C 1909+72 likely shares the same node or filament as the signpost active galactic nuclei (AGN), but it is not detected in ${ }^{12} \mathrm{CO}$ despite $\sim 20000 \mathrm{~km} \mathrm{~s}^{-1}$ of velocity coverage. Also in the 6C 1909+72 field, a large, red dust feature spanning $\approx 500 \mathrm{kpc}$ is aligned with the radio jet. We suggest several processes by which metal-rich material may have been transported, favouring a collimated outflow reminiscent of the jet-oriented metal enrichment seen in local cluster environments. Our interferometric imaging reveals a gas-rich companion to $\mathrm{B} 3 \mathrm{~J} 2330+3927$; indeed, all bar one of the eight $z \gtrsim 2$ radio galaxies (or companions) detected in ${ }^{12} \mathrm{CO}$ provide some evidence that starburst activity in radio-loud AGN at high redshift is driven by the interaction of two or more gas-rich systems in which a significant mass of stars has already formed, rather than via steady accretion of cold gas from the cosmic web. We find that the ${ }^{12} \mathrm{CO}$ brightness temperature ratios in radio-loud AGN host galaxies are significantly higher than those seen in similarly intense starbursts where AGN activity is less pronounced. Our most extreme example, where $L_{\mathrm{CO} 4-3}^{\prime} / L_{\mathrm{CO} 1-0}^{\prime}>2.7$, provides evidence that significant energy is being deposited rapidly into the molecular gas via X-rays and/or mechanical ('quasar-mode') feedback from the AGN, leading to a high degree of turbulence
\end{abstract}

${ }^{\star}$ E-mail: rji@roe.ac.uk 
globally and a low optical depth in ${ }^{12} \mathrm{CO}$ - feedback that may lead to the cessation of star formation on a time-scale commensurate with that of the jet activity, $\lesssim 10 \mathrm{Myr}$.

Key words: galaxies: active - galaxies: high-redshift - galaxies: starburst - infrared: galaxies - radio lines: galaxies.

\section{INTRODUCTION}

High-redshift radio galaxies $\left(\mathrm{H}_{z} \mathrm{RGs}\right)$ are typically identified via their ultrasteep-spectrum radio emission $\left(\alpha<-1\right.$ where $\left.S_{v} \propto v^{\alpha}\right)$ in flux-limited surveys (e.g. Tielens, Miley \& Willis 1979; Röttgering et al. 1994). Despite the inevitable and extreme youth of the radio jets that draw our attention to these distant galaxies, they are associated with the most massive stellar populations of any known galaxy class - and presumably the most massive black holes and host galaxies - out to the highest redshifts (Best, Longair \& Röttgering 1998; Blundell \& Rawlings 1999; Seymour et al. 2007).

In the submillimetre (submm; rest-frame far-infrared, FIR) regime, $\mathrm{H} z \mathrm{RGs}$ were first explored using relatively primitive submm detectors such as UKT14 on the James Clerk Maxwell Telescope (e.g. Dunlop et al. 1994). Later, with the advent of sensitive submm cameras, their submm luminosities (and hence dust masses) were found to be a strongly increasing function of redshift (Archibald et al. 2001; Reuland et al. 2004), even beyond the peak epoch of activity for submm galaxies (SMGs, $z \sim 1-3$; Chapman et al. 2005).

As rare, massive systems, HzRGs are often employed as signposts to what are expected - based on our understanding of how cosmic structures form and evolve (e.g. Davis et al. 1985) - to be overdense regions of the early Universe. Surveys found excesses of various galaxy types around $\mathrm{H}_{z} \mathrm{RG}$, including Lyman-break galaxies, Ly $\alpha$ emitters and SMGs (Ivison et al. 2000; Miley et al. 2004; Greve et al. 2007; Venemans et al. 2007; Overzier et al. 2008; Hatch et al. 2011). Stevens et al. (2003) presented submm imaging of seven $\mathrm{HzRGs}$, several of which appear to contain extended ( 520 arcsec, $\sim 35-150 \mathrm{kpc}$ ) dust emission, co-spatial with similarly extended ultraviolet (UV) emission in several cases (Hippelein \& Meisenheimer 1993; Hatch et al. 2008). This suggests that obscured starbursts in these overdense regions at $z \gtrsim 3$ may differ from the compact ( $\lesssim 0.5$ arcsec or $\lesssim 4 \mathrm{kpc}$ ) events seen in local ultraluminous IR galaxies (ULIRGs) and, indeed, as seen by high-resolution ${ }^{12} \mathrm{CO}$ and radio continuum imaging of the general $z \sim 2$ SMG field population (Biggs \& Ivison 2008; Tacconi et al. 2008; Younger et al. 2008). From this we might conclude that the mechanism for the formation of the very massive galaxies in these overdense regions may be fundamentally different to that of the $\gtrsim L_{\star}$ galaxies forming from SMGs (Smail et al. 2004), although the earliest ${ }^{12} \mathrm{CO} J=1-0$ imaging of SMGs (e.g. Carilli et al. 2011; Hodge et al. 2011; Ivison et al. 2011; Riechers et al. 2011a,b) using the Karl Jansky Very Large Array (JVLA) (Perley et al. 2011) suggests that their cold gas may be more extended than the high- $J{ }^{12} \mathrm{CO}$ tracers employed previously.

Valiant, early attempts to detect ${ }^{12} \mathrm{CO}$ towards $\mathrm{Hz}$ RGs with singledish telescopes ended in failure (e.g. Evans et al. 1996; van Ojik et al. 1997), but the stable spectral baselines afforded by interferometers eventually allowed the secure detection of ${ }^{12} \mathrm{CO} J=3-2$ towards 53W002 at $z=2.39$ (Scoville et al. 1997; Alloin, Barvainis \& Guilloteau 2000) and ${ }^{12} \mathrm{CO} J=4-3$ towards $4 \mathrm{C} 60.07$ at $z=3.79$ and 6C 1909+72 at $z=3.53$ (Papadopoulos et al. 2000, hereafter P00), B3 2330+3927 at $z=3.09$ (De Breuck et al. 2003a, hereafter
DB03), TN J0121+1320 at $z=3.52$ (De Breuck, Neri \& Omont 2003b) and 4C 41.17 at $z=3.80$ (De Breuck et al. 2005). Detections of the ${ }^{12} \mathrm{CO} J=1-0$ line - that most sensitive to cold gas (Papadopoulos \& Ivison 2002) - are particularly difficult towards radio galaxies: synchrotron emission often dominates at the frequency of the line, $\sim 115 \mathrm{GHz}$, which is expected to be roughly an order of magnitude fainter than ${ }^{12} \mathrm{CO} J=4-3$. The robust detection of ${ }^{12} \mathrm{CO} J=1-0$ by Emonts et al. (2011) towards MRC 0152-209 is the exception that proves the rule, albeit at a modest redshift $(z=$ 1.92). More often, $J=1-0$ has been proved to be elusive (Ivison et al. 1996; Papadopoulos et al. 2005) or detections have been of tentative nature (e.g. Greve, Ivison \& Papadopoulos 2004; Klamer et al. 2005).

Here, we present new observations of two $\mathrm{H}_{z} \mathrm{RGs}$, 6C 1909+72 (also known as 4C 72.26 and TXS J1908+7220) and B3 J2330+3927 (Pentericci et al. 2000, DB03) using JVLA, the Institut de Radioastronomie Millimétrique's Plateau de Bure Interferometer (IRAM PdBI) and Herschel ${ }^{1}$ (Pilbratt et al. 2010). The PACS and SPIRE instruments (Griffin et al. 2010; Poglitsch et al. 2010) aboard Herschel offer unprecedented sensitivity, with resolution well-matched to ground-based predecessors such as SCUBA (Holland et al. 1999). They cover the decade of wavelengths from 70 to $500 \mu \mathrm{m}$ with $\lambda / \Delta \lambda \sim 3$, which allows us to probe the peak of the spectral energy distribution (SED) of a dusty galaxy out to $z \sim 4$, thereby determining its star formation rate (SFR, via its FIR luminosity, $L_{\mathrm{IR}}$, across rest-frame $8-1000 \mu \mathrm{m}$, e.g. Kennicutt 1998) and its characteristic dust temperature, $T_{\mathrm{d}}$. Beyond the local Universe, these quantities have only rarely been well constrained until now. Alternatively, we can adopt a reasonable value for $T_{\mathrm{d}}$ and then estimate the redshifts of the SMGs seen in the vicinity of $\mathrm{H}_{z} \mathrm{RG}$ signposts (e.g. Stevens et al. 2003) using their rest-frame FIR colours (e.g. Eales et al. 2003; Greve et al. 2008; Penner et al. 2011), i.e. without resorting to conventional spectroscopy, thereby determining the likelihood that they inhabit a massive structure alongside the HzRG.

In the next section, we describe an extensive set of observations, and then present the reduced images, spectra and associated analysis in Section 3. Section 4 contains our interpretation of those data and discussion of their implications. We finish with our conclusions in Section 5. Throughout the paper, we use a cosmology with $H_{0}=$ $71 \mathrm{~km} \mathrm{~s}^{-1} \mathrm{Mpc}^{-1}, \Omega_{\mathrm{m}}=0.27$ and $\Omega_{\Lambda}=0.73$.

\section{OBSERVATIONS}

The observations described hereafter targeted the $\mathrm{H}_{z} \mathrm{RGs}$, 6C 1909+72 and B3 J2330+3927. The former presents a classic double-lobed (plus core) radio morphology, subtending $\sim 15$ arcsec, and has a total 1.4-GHz flux density, $S_{1.4 \mathrm{GHz}} \sim 259 \mathrm{mJy}$; the latter

\footnotetext{
${ }^{1}$ Herschel is an ESA space observatory with science instruments provided by European-led Principal Investigator consortia and with important participation from NASA.
} 
is brighter, $S_{1.4 \mathrm{GHz}} \sim 405 \mathrm{mJy}$, with what appears at first sight to be a similar morphology, if more compact ( $\sim 2 \operatorname{arcsec})$, but whose high-resolution radio imaging revealed to be an unusually onesided jet driven by a compact, flat-spectrum core (Pérez-Torres \& De Breuck 2005). Alongside PKS 1138-262, 6C 1909+72 and B3 J2330+3927 are the most luminous of the $69 \mathrm{HzRG}$ explored by Seymour et al. (2007) in the rest-frame near-IR using Spitzer.

\subsection{JVLA observations}

Whilst the JVLA was in its most compact configuration during 2011 September-November, we acquired, amongst the first data taken in a new mode offering almost an order of magnitude more bandwidth than was previously possible, tuning to the ${ }^{12} \mathrm{CO} J=$ 1-0 transition at $115.27120256 \mathrm{GHz}$ (Morton \& Noreau 1994) for our target $\mathrm{H}_{z}$ RGs.

Short slots, usually 2-3h long, were scheduled dynamically to ensure excellent phase stability and transparency in the $\mathrm{K}$ and $\mathrm{Ka}$ atmospheric windows for 6 C $1909+72$ and B3 J2330+392, respectively. During these slots, data were recorded ${ }^{2}$ every $3 \mathrm{~s}$ in each of $2 \times 8$ contiguous baseband (dual-polarization) pairs, each baseband comprising $64 \times 2 \mathrm{MHz}$ channels for a total dual-polarization bandwidth of $2048 \mathrm{MHz}$, well over $20000 \mathrm{~km} \mathrm{~s}^{-1}$ at the redshift of 6C 1909+72. Bright, compact calibration sources - flat-spectrum blazars lying within a few degrees of our target galaxies - were observed every few minutes to determine accurate complex gain solutions and bandpass corrections. 3C 48 and 3C 286 were also observed to set the absolute flux scale, and the pointing accuracy was checked locally every hour.

For 6C 1909+72 the two sets of eight contiguous baseband pairs were themselves placed contiguously. We shifted the ${ }^{12} \mathrm{CO} J=1-0$ line (expected at $25.434 \mathrm{GHz}$ for $z=3.5322$ ) by $180 \mathrm{MHz}$ from the centre of the available bandwidth to avoid the edge of a baseband, or one of the end basebands.

For B3 J2330+3927, the ${ }^{12} \mathrm{CO} J=1-0$ line lies below $32 \mathrm{GHz}$ in the $K a$ band. As such, only one set of eight contiguous baseband pairs could be deployed on the line (expected at $28.156 \mathrm{GHz}$ for $z=3.094$, but purposefully offset by $64 \mathrm{MHz}$ to avoid the edge of a baseband); the other set of eight basebands were tuned to $32.5 \mathrm{GHz}$.

The data were reduced and imaged using AIPS following the recipes described by Ivison et al. (2011), though with a number of significant changes: data were loaded using BDF2AIPS, avoiding any compression, and FRING was used to optimize the delays, in software, based on $1 \mathrm{~min}$ of data for $3 \mathrm{C} 48$ or $3 \mathrm{C} 286$. The basebands were knitted together using the NOIFS task, yielding $u v$ data sets with either 1024 or $512 \times 2 \mathrm{MHz}$ channels. Continuum images were made using all of these data to obtain very deep maps of the synchrotron emission at 25.4 and $28.2 \mathrm{GHz}$ (for 6C 1909+72 and $\mathrm{B} 3 \mathrm{~J} 2330+3927$, respectively), which were then cleaned and subtracted from the $u v$ data (using UVSUB). The channels were then imaged in groups of four (yielding $\sim 90 \mathrm{~km} \mathrm{~s}^{-1}$ velocity resolution) over $\sim 3$-arcmin-diameter fields, with natural weighting (ROBUST $=5$ ), to form large cubes centred on the radio galaxies. The resulting spatial resolutions were $4.0 \times 3.0 \operatorname{arcsec}^{2}$ at position angle (PA), $171^{\circ}$, for $6 \mathrm{C} 1909+72$ and $3.4 \times 2.8 \operatorname{arcsec}^{2}$ at PA, $72^{\circ}$, for B3 J2330+3927.

\footnotetext{
${ }^{2} \mathrm{~A}$ bug in the correlator back end computer during this period resulted in only $1 \mathrm{~s}$ of data being saved per integration, meaning that a significant fraction of the useful data were lost.
}

\subsection{IRAM PdBI imaging}

Observations of $6 \mathrm{C} 1909+72$ were made in ${ }^{12} \mathrm{CO} J=4-3$ using PdBI's $C$ and $D$ configurations in the 3-mm atmospheric window during 2007 July (with five antennas) and December (with six antennas) in a series of $\sim 6$ - to 11 -h tracks with good phase stability (0.6-1.0 arcsec seeing) and transparency (1.5-2.5 $\mathrm{mm}$ of water vapour). The local calibrator, $1928+738$, was used to track amplitude and phase, with $1642+690$ used for an independent check. Absolute fluxes were calibrated using MWC 349. Data cubes were made via AIPS using a natural weighting scheme, yielding a $3.1 \times$ $2.7 \operatorname{arcsec}^{2}$ beam with the major axis at a PA of $47^{\circ}$.

The original PdBI data acquired by DB03 for B3 J2330+3927 were re-imaged via AIPS using a natural weighting scheme, discarding those channels observed only in the compact configuration, yielding a $3.2 \times 2.4 \operatorname{arcsec}^{2}$ beam with the major axis at a PA of $72^{\circ}$.

\subsection{Herschel imaging}

The Herschel data presented in this paper comprise a small part of the Herschel Radio Galaxy Evolution Project OT1 programme (Seymour et al. 2012). With PACS, we made near-orthogonal scans of our $\mathrm{H} z \mathrm{RG}$ targets at nominal speed (i.e. with the telescope tracking at $20 \operatorname{arcsec~s}^{-1}$ ) using mini-map mode, spending a total of $288 \mathrm{~s}$ on source, recording data simultaneously at 100 and $160 \mu \mathrm{m}$, each scan comprising $12 \times 4$ arcmin scan legs separated by 4 arcsec. With SPIRE we approached the confusion limit (Nguyen et al. 2010) with just three repetitions (111 s on source) of its 'small map' mode, obtaining data simultaneously at 250, 350 and $500 \mu \mathrm{m}$. The SPIRE images were produced using the standard pipeline; the PACS data were tackled with a variant of the pipeline developed by Ibar et al. (2010)

Flux densities for SPIRE were measured using SUSSEXTRACTOR (Smith et al. 2012), as implemented in the Herschel Data Processing System. For PACS, we used apertures of radii 9 and 13.8 arcsec at 100 and $160 \mu \mathrm{m}$, respectively, with appropriate aperture corrections, with errors determined by placing many random apertures in regions of the image with integration times similar to those of our targets.

\section{RESULTS}

\section{$3.16 C 1909+72$}

The synchrotron continuum emission from 6C 1909+72 dominates the observed flux density at $25 \mathrm{GHz}$, being almost an order of magnitude brighter than the ${ }^{12} \mathrm{CO}$ line peak (Fig. 1, left). To assess the ${ }^{12} \mathrm{CO}$ line properties we subtracted an accurate model of the continuum emission, exploiting the impressive bandwidth now available at JVLA.

In the resulting continuum-free JVLA data cube, the ${ }^{12} \mathrm{CO} J=$ $1-0$ line emission is seen clearly, centred at $z=3.5324 \pm 0.0007$ (Fig. 1, right), closely matching that seen in our ${ }^{12} \mathrm{CO} J=4-3$ spectrum from IRAM PdBI, $z=3.5324 \pm 0.0006$. The basic characteristics of the ${ }^{12} \mathrm{CO}$ line emission - peak and total flux densities, line widths, etc. - are listed in Table 1 , for both transitions. It is notable that we measure $I_{\mathrm{CO} 4-3}=2.69 \pm 0.27 \mathrm{Jy} \mathrm{km} \mathrm{s}^{-1}$, cf. the $1.62 \pm 0.30 \mathrm{Jy} \mathrm{km} \mathrm{s}^{-1}$ measured by $\mathrm{P} 00$, who may also have underestimated the line width due to a lack of available bandwidth.

Both the ${ }^{12} \mathrm{CO} J=1-0$ and $J=4-3$ emissions are partially resolved, even with a natural weighting scheme. Some velocity 

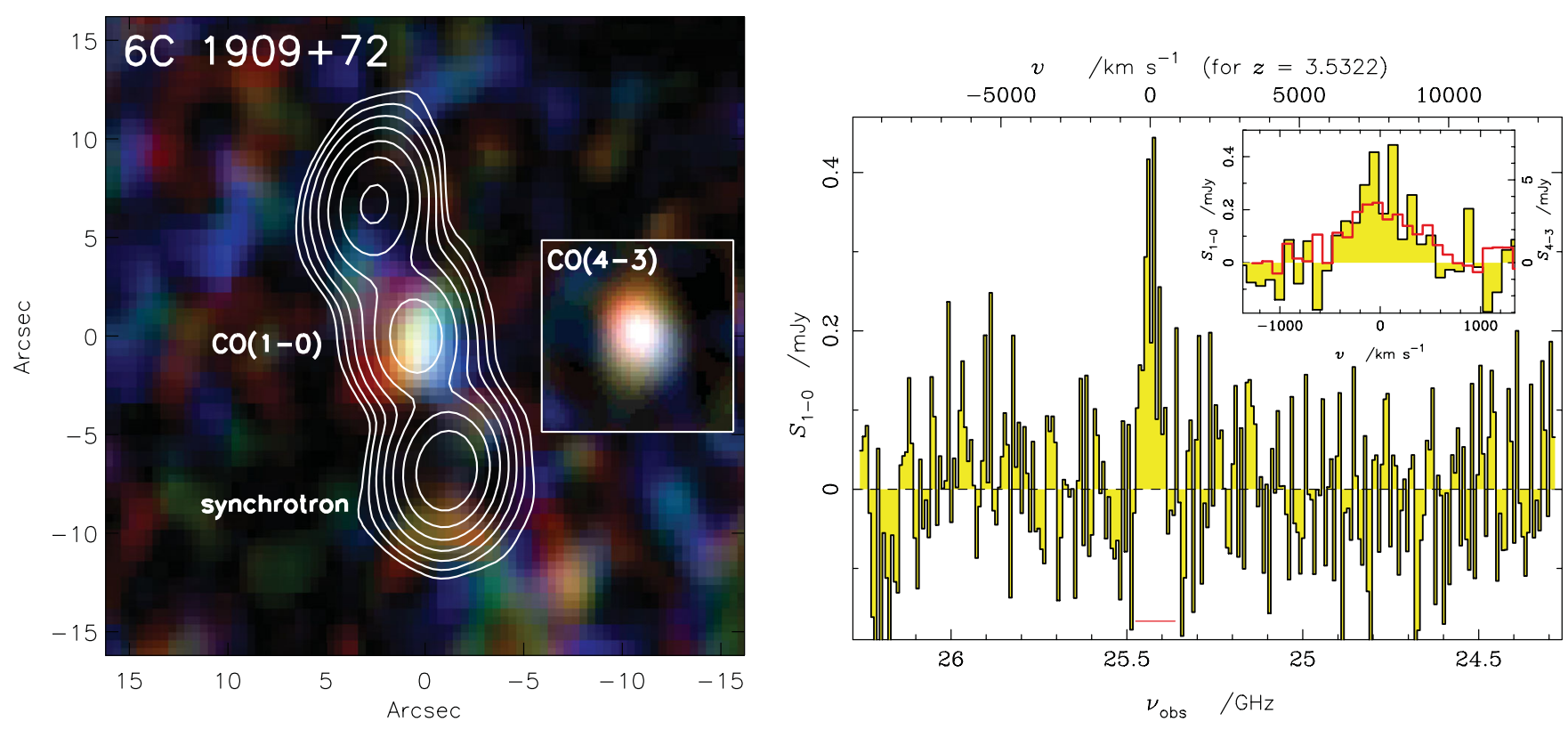

Figure 1. Left: false colour image of the ${ }^{12} \mathrm{CO} J=1-0$ emission from $6 \mathrm{C} 1909+72$, as measured by JVLA. $\mathrm{N}$ is up; $\mathrm{E}$ is left. Inset: ${ }^{12} \mathrm{CO} J=4-3$ as measured at IRAM PdBI, on the same spatial scale. Emission coming towards and going away from us relative to the systemic velocity is represented with the appropriate colours, revealing hints of a velocity field common to both ${ }^{12} \mathrm{CO}$ lines, though not globally. The much brighter $25-\mathrm{GHz}$ synchrotron emission, against which we have struggled to discern the ${ }^{12} \mathrm{CO} J=1-0$ emission, is represented with isophotal contours at $-3,3,6,12, \ldots \times$ the local noise level. Right: ${ }^{12} \mathrm{CO} J=1-0$ spectrum of $6 \mathrm{C} 1909+72$, after subtraction of the dominant synchrotron emission component, smoothed with a $140 \mathrm{~km} \mathrm{~s}^{-1}$ full width at half-maximum (FWHM) Gaussian. Inset: zoomed in on the line, with the new IRAM PdBI ${ }^{12} \mathrm{CO} J=4-3$ spectrum (Section 2.2) shown in red, binned to $100 \mathrm{~km} \mathrm{~s}^{-1}$ and scaled by $16^{-1} \times$ to be on the same Rayleigh-Jeans brightness temperature $\left(T_{\mathrm{b}}\right)$ scale as the ${ }^{12} \mathrm{CO} J=1-0$ spectrum. The velocity scales correspond to $z=3.5322$, approximately midway between the two components ( $z=3.5203$ and 3.5401) identified by Smith et al. (2010). The red horizontal line shows the spectral region summed to create the ${ }^{12} \mathrm{CO} J=1-0$ image shown alongside, and to determine $I_{\mathrm{CO}}$.

Table 1. ${ }^{12} \mathrm{CO}$ emission-line properties.

\begin{tabular}{|c|c|c|c|c|c|c|}
\hline Target & $\begin{array}{c}\text { RA } \\
(\mathrm{J} 2000)\left({ }^{h . m . s}\right)\end{array}$ & $\begin{array}{c}\text { Dec. } \\
(\mathrm{J} 2000)\left({ }^{\circ}:^{\prime}:^{\prime \prime}\right)\end{array}$ & $\begin{array}{c}\text { Peak } S_{v} \\
(\mathrm{mJy})\end{array}$ & $z$ & $\begin{array}{l}\text { FWHM } \\
\left(\mathrm{km} \mathrm{s}^{-1}\right)\end{array}$ & $\begin{array}{c}I_{\mathrm{CO}} \\
\left(\mathrm{Jy} \mathrm{km} \mathrm{s}^{-1}\right)\end{array}$ \\
\hline \multicolumn{7}{|l|}{$J=1-0$} \\
\hline B3 J2330+3927 (AGN host) & - & - & $3 \sigma<0.28$ & - & - & $3 \sigma<0.074$ \\
\hline JVLA J233024.69+392708.6 (c) & $23: 30: 24.69 \pm 0.02$ & $+39: 27: 08.58 \pm 0.16$ & $0.21 \pm 0.04$ & $3.0884 \pm 0.0010$ & $720 \pm 170$ & $0.162 \pm 0.034$ \\
\hline \multicolumn{7}{|l|}{$J=4-3:$} \\
\hline JVLA J233024.69+392708.6 (c) & $23: 30: 24.62 \pm 0.02$ & $+39: 27: 08.46 \pm 0.18$ & $1.9 \pm 0.4$ & $3.0901 \pm 0.0006$ & $520 \pm 110$ & $1.12 \pm 0.17$ \\
\hline
\end{tabular}

structure can be discerned in Fig. 1, common to both transitions, but there does not appear to be a strong, coherent gradient.

From the measured ${ }^{12} \mathrm{CO}$ properties, we find $L_{\mathrm{CO} 1-0}^{\prime}=(1.19 \pm$ $0.26) \times 10^{11} \mathrm{~K} \mathrm{~km} \mathrm{~s}^{-1} \mathrm{pc}^{2}$ and the molecular gas mass, $M_{\text {gas }}$ $\left(\mathrm{H}_{2}+\mathrm{He}\right)$, is therefore likely around $\sim 10^{11} \mathrm{M}_{\odot}$, naively adopting $M_{\text {gas }} / L_{\mathrm{CO}}^{\prime}=0.8 \mathrm{M}_{\odot}\left(\mathrm{K} \mathrm{km} \mathrm{s}^{-1} \mathrm{pc}^{2}\right)^{-1}$ (Downes \& Solomon 1998).

\subsection{B3 J2330+3927}

At the position of the radio galaxy core, as identified by PérezTorres \& De Breuck (2005) and detected in ${ }^{12} \mathrm{CO} J=4-3$ using PdBI by DB03, we find synchrotron emission $\left(S_{28.2 \mathrm{GHz}}=5.14 \pm\right.$ $0.02 \mathrm{mJy}$ centred at $23^{\mathrm{h}} 30^{\mathrm{m}} 24 \mathrm{~s} 866,+39^{\circ} 27^{\prime} 11^{\prime \prime} .84 \mathrm{~J} 2000$ ) but there is no evidence of the ${ }^{12} \mathrm{CO} J=1-0$ emission in a synchrotronsubtracted data cube. The $(3 \sigma)$ limit we can set, assuming the same line width as the ${ }^{12} \mathrm{CO} J=4-3$ emission $\left(830 \mathrm{~km} \mathrm{~s}^{-1} \mathrm{FWHM}\right)$ and following appendix A of Seaquist, Ivison \& Hall (1995), is $I_{\mathrm{CO}}<$ $0.074 \mathrm{Jy} \mathrm{km} \mathrm{s}^{-1}$. The basic characteristics of the ${ }^{12} \mathrm{CO}$ line emission are again listed in Table 1, for both transitions.

Fig. 2 shows the Keck/NIRC $K$-band imaging (from DB03) as a grey-scale, superimposed with contours to show the emission in 8.4-GHz continuum (synchrotron), ${ }^{12} \mathrm{CO} J=1-0$ and ${ }^{12} \mathrm{CO} J=$ 4-3. Approximately 4 arcsec to the SSW of the active galactic nuclei (AGN) (and $\sim 260 \mathrm{~km} \mathrm{~s}^{-1}$ bluewards of its ${ }^{12} \mathrm{CO} J=4-3$ redshift) we do see emission in ${ }^{12} \mathrm{CO} J=1-0$. It is coincident with what DB03 labelled component ' $c$ ' and tentatively suggested might lie at the same redshift as the radio galaxy. We show its spectra in ${ }^{12} \mathrm{CO} J=1-0$ and $J=4-3$ in Fig. 2 . Our new ${ }^{12} \mathrm{CO} J=1-0$ imaging and our re-analysis of the ${ }^{12} \mathrm{CO} J=4-3$ data make it clear that component $\mathrm{c}$ is in fact a gas-rich galaxy in the immediate vicinity of the radio-loud AGN, which is presumably interacting with (and thereby triggering activity in) the host galaxy of the AGN. Component $\mathrm{c}$ is detected in at least three IRAC bands and so has 

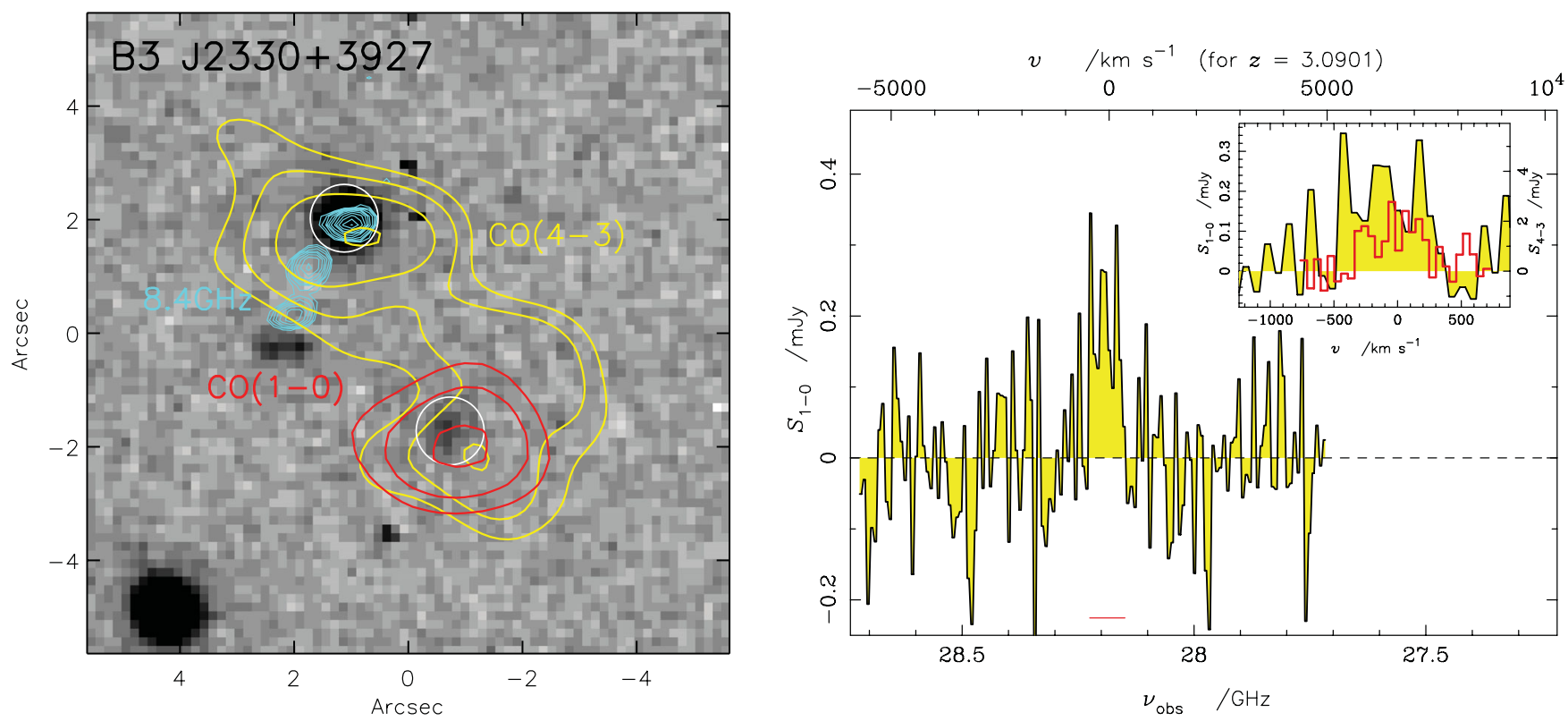

Figure 2. Left: grey-scale $K^{\prime}$ image of the B3 J2330+3927 field (DB03) with isophotal contours of the ${ }^{12} \mathrm{CO} J=1-0$ emission in red $(-3,3,3 \sqrt{2}, 6, \ldots \times$ local noise level). $\mathrm{N}$ is up; $\mathrm{E}$ is left. The ${ }^{12} \mathrm{CO} J=1-0$ is associated with component $\mathrm{c}$ (circled, lower-right), rather than the core of the AGN host galaxy (also circled, betrayed by its powerful synchrotron emission - cyan contours - Pérez-Torres \& De Breuck 2005). The ${ }^{12} \mathrm{CO} J=4-3$ emission is shown in yellow a radically different morphology from that presented by DB03. The brightest component is centred on the AGN while a fainter clump lies to the south-west, coincident with component $\mathrm{c}$. The astrometric uncertainties here are $\lesssim 0.5 \mathrm{arcsec}$. Right: ${ }^{12} \mathrm{CO} J=1-0$ spectrum of JVLA 233024.69+392708.6 (component c), near B3 J2330+3927. Inset: zoomed in on the line, with the IRAM PdBI ${ }^{12} \mathrm{CO} J=4-3$ spectrum of the same galaxy (Section 2.2) shown in red, binned to $53 \mathrm{~km} \mathrm{~s}^{-1}$ and scaled by $16^{-1} \times$ to be on the same Rayleigh-Jeans $T_{\mathrm{b}}$ scale as the ${ }^{12} \mathrm{CO} J=1-0$ spectrum. The red horizontal line shows the spectral region summed to create the ${ }^{12} \mathrm{CO} J=1-0$ image shown alongside, and to determine $I_{\mathrm{CO}}$.

a significant stellar population. It is not obvious that it contributes significantly to the Lyman $\alpha$ halo that surrounds B3 J2330+3927 (Matsuda et al. 2009).

Fig. 3 reveals the dynamical structure of the ${ }^{12} \mathrm{CO}$ emission, with a blue-red gradient leading from component $\mathrm{c}$ to beyond the radioloud AGN. The continuum emission at $\lambda_{\text {obs }}=160,250$ and $850 \mu \mathrm{m}$ (rest-frame 40, 60 and $200 \mu \mathrm{m}$ ) seen by PACS, SPIRE and SCUBA is also shown. At $\lambda_{\mathrm{obs}}=24 \mu \mathrm{m}$, the emission is predominantly from the AGN; at $\lambda_{\mathrm{obs}}=100$ and $160 \mu \mathrm{m}$, it is predominantly from component $\mathrm{c}$; and at $\lambda_{\text {obs }} \geq 250 \mu \mathrm{m}$ the emission is centred between the two. We have evidence, then, that dust at a variety of temperatures is distributed throughout the system, yet we lack the spatial resolution to disentangle these components reliably.

The total flux in ${ }^{12} \mathrm{CO} J=1-0$ for component $\mathrm{c}$ is $I_{\mathrm{CO}}=0.162 \pm$ $0.034 \mathrm{Jy} \mathrm{km} \mathrm{s}^{-1}$. The excitation situation for component $\mathrm{c}$ is comparable to that of SMGs (Harris et al. 2010; Ivison et al. 2011), with a brightness temperature $\left(T_{\mathrm{b}}\right)$ ratio, $L_{\mathrm{CO} 4-3}^{\prime} / L_{\mathrm{CO} 1-0}^{\prime}=r_{4-3 / 1-0}=$ $0.43 \pm 0.11$ (cf. $0.41 \pm 0.07-$ Bothwell et al. 2012). This differs strikingly from that of the radio galaxy core, where the $T_{\mathrm{b}}$ ratio is entirely inconsistent with thermal emission, $r_{4-3 / 1-0}>2.7(3 \sigma)$, i.e. the emission is superthermal ${ }^{3}$ (see Section 4.1).

DB03 assumed $r_{4-3 / 1-0}=0.45$ to extrapolate their ${ }^{12} \mathrm{CO} J=$ 4-3 measurement to $J=1-0$ and calculate a gas mass for B3 $\mathrm{J} 2330+3927$. We can see now that this will have resulted in an overestimate of $M_{\text {gas }}$ for the AGN host galaxy. Our limit on $I_{\mathrm{CO} 1-0}$

\footnotetext{
${ }^{3}$ Strictly, superthermal excitation exists only where local thermodynamic equilibrium has been violated by a population inversion. Here, we use it as shorthand for $r>1$.
}

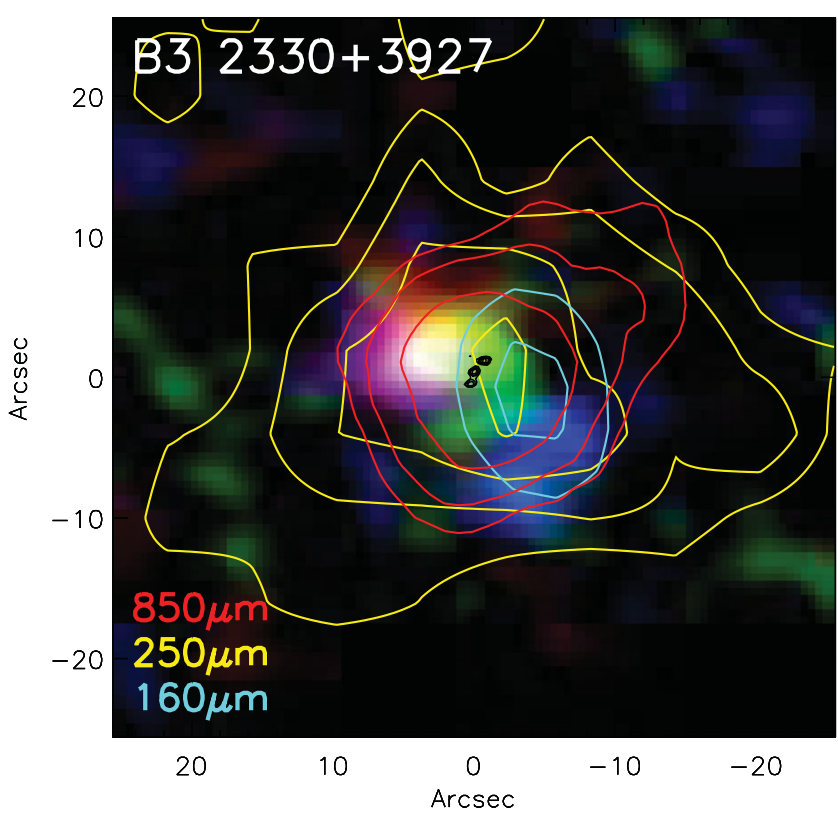

Figure 3. Three velocity slices in ${ }^{12} \mathrm{CO} J=4-3$ towards B $3 \mathrm{~J} 2330+3927$ displayed as a false-colour RGB image, showing how the superthermal emission centred near the radio-loud AGN (shown as black dots) is offset to the red from the ${ }^{12} \mathrm{CO}$ emission associated with the neighbouring, gas-rich galaxy, JVLA 233024.69+392708.6. Cyan, yellow and red contours (starting at $3 \sigma$ and spaced by $\sqrt{2}$ ) show the $160-, 250-$ and $850-\mu \mathrm{m}$ continuum emission as seen by Herschel and SCUBA (Stevens et al. 2003), with no astrometric tweaks applied, consistent with cold dust distributed throughout the system. $\mathrm{N}$ is up; $\mathrm{E}$ is left. 
Table 2. Measured flux densities and properties of $\mathrm{HzRG}$ and brightest field SMGs.

\begin{tabular}{lcccccc}
\hline Target & $\begin{array}{c}S_{100 \mu \mathrm{m}} \\
(\mathrm{mJy})\end{array}$ & $\begin{array}{c}S_{160 \mu \mathrm{m}} \\
(\mathrm{mJy})\end{array}$ & $\begin{array}{c}S_{250 \mu \mathrm{m}}^{a} \\
(\mathrm{mJy})\end{array}$ & $\begin{array}{c}S_{350 \mu \mathrm{m}}^{a} \\
(\mathrm{mJy})\end{array}$ & $\begin{array}{c}S_{500 \mu \mathrm{m}}^{a} \\
(\mathrm{mJy})\end{array}$ & $\begin{array}{c}S_{850 \mu \mathrm{m}} \\
(\mathrm{mJy})\end{array}$ \\
\hline 6C 1909+72 & $17.0 \pm 3.0$ & $34.9 \pm 6.5$ & $57.2 \pm 2.7$ & $69.6 \pm 2.8$ & $63.4 \pm 3.3$ & $34.9 \pm 3.0$ \\
SMM J190827.5+721928 & $0.0 \pm 2.9$ & $-0.1 \pm 6.7$ & $45.1 \pm 2.6$ & $65.1 \pm 2.7$ & $61.2 \pm 3.0$ & $23.0 \pm 2.5$ \\
B3 J2330+3927 & $11.3 \pm 2.9^{b}$ & $27.0 \pm 6.2^{b}$ & $52.0 \pm 2.7$ & $60.3 \pm 2.7$ & $57.3 \pm 3.3$ & $22.2 \pm 2.7$ \\
SMM J233019.1+392703 & $5.4 \pm 3.3$ & $4.2 \pm 11.9$ & $31.6 \pm 2.8$ & $35.4 \pm 2.6$ & $21.1 \pm 3.4$ & $8.2 \pm 1.9$ \\
\hline
\end{tabular}

${ }^{a}$ Uncertainty does not include the contribution from confusion noise, nor any possible systematic offset in flux calibration.

${ }^{b}$ Astrometry is consistent with a significant fraction of the PACS emission originating from JVLA 233024.69+392708.6 (Fig. 3).

implies that $L_{\mathrm{CO}}^{\prime}<2.9 \times 10^{10} \mathrm{~K} \mathrm{~km} \mathrm{~s}^{-1} \mathrm{pc}^{2}$ and $M_{\text {gas }}<2.3 \times$ $10^{10} \mathrm{M}_{\odot}$ where $M_{\text {gas }} / L_{\mathrm{CO}}^{\prime}=0.8 \mathrm{M}_{\odot}\left(\mathrm{K} \mathrm{km} \mathrm{s}^{-1} \mathrm{pc}^{2}\right)^{-1}$.

For the gas-rich galaxy previously known as component $\mathrm{c}$, which we rename JVLA $233024.69+392708.6$, we find $L_{\mathrm{CO}}^{\prime}=(6.9 \pm$ $1.5) \times 10^{10} \mathrm{~K} \mathrm{~km} \mathrm{~s}^{-1} \mathrm{pc}^{2}$ and $M_{\text {gas }} \sim 5.5 \times 10^{10} \mathrm{M}_{\odot}$.

\subsection{On the HzRGs and their environments}

The overdensity of bright SMGs found around $\mathrm{Hz}$ RGs, their faintness at optical/IR wavebands and the alignment of those SMGs with the jets from the central AGN were taken as tentative evidence that $\mathrm{H}_{z} \mathrm{RG}$ are signposting massive structures in which other galaxies are also undergoing intense starbursts (Ivison et al. 2000; Stevens et al. 2003).

Our deep Herschel imaging allows us to assess the likely redshifts of these bright neighbouring SMGs via their FIR/submm colours (e.g. Amblard et al. 2010, though such colours are only sensitive to $(1+z) / T_{\mathrm{d}}-$ see Blain 1999). We report their FIR/submm flux densities in Table 2 .

The upper panels of Fig. 4 show two of the colour-colour diagnostic plots employed by Amblard et al. (2010) to assess the redshift and $T_{\mathrm{d}}$ of galaxies detected by H-ATLAS (Eales et al. 2010), probing their colours across the rest-frame $\sim 100-\mu \mathrm{m}$ bump. The coloured backgrounds indicate the redshifts of model SEDs. The new, lower panel of Fig. 4 is based on the same model SEDs and is designed to exploit information from our SCUBA $850-\mu$ m imaging that is particularly relevant to galaxies at $z>2$.

The red crosses represent B3 J2330+3927 and its brightest SMG neighbour, SMM J233019.1+392703. The position of the latter particularly in the lower two plots - strongly suggests that it lies at a lower redshift than the radio galaxy, nearer the peak of the radio-detected SMG population at $z \sim 2.2$ (e.g. Chapman et al. 2005).

The black crosses represent 6C $1909+72$ and the very bright, nearby SMG, SMMJ190827.5+721928. Their rest-frame FIR colours are remarkably similar in the lower two colour-colour plots. The two galaxies differ in $S_{160} / S_{250}$, i.e. the colours on the Wien side of their SEDs. The limit for the bright companion is consistent with the known redshift of the radio galaxy, but the colour of the radio galaxy is bluer than we might expect. On balance, the evidence is consistent with the proposition that these two dusty starbursts share the same node or filament ${ }^{4}$ of the cosmic web.

With $\Delta v>10000 \mathrm{~km} \mathrm{~s}^{-1}$ of velocity coverage available to us via the WIDAR correlator at JVLA, we should be sensitive to the ${ }^{12} \mathrm{CO}$

\footnotetext{
${ }^{4}$ A typical filament at $z \sim 3.5$ spans of the order of $\approx 30 h^{-1} \mathrm{Mpc}$ (e.g. Springel et al. 2005a). SMM J190827.5+721928 is separated by 45 arcsec $(335 \mathrm{kpc})$ in the plane of the sky from 6C $1909+72$. It would need to lie within $\delta z \lesssim 0.04$ to inhabit the same sheet or filament.
}

$J=1-0$ emission from bright SMGs in our target fields, should they have a similar ratio of $S_{\mathrm{CO}} / S_{850 \mu \mathrm{m}}$ and if - as seems likely, at least for 6C 1909+72 and SMMJ190827.5+721928 - they lie in the same cosmic structure as the central $\mathrm{H}_{z} \mathrm{RG}$.

We do not detect the ${ }^{12} \mathrm{CO} J=1-0$ emission from SMM J233019.1+392703 in the B3 J2330+3927 field. However, this SMG is less than half as bright as the nearby $\mathrm{H}_{z} \mathrm{RG}$; its emission is attenuated severely ( 82 per cent) by the JVLA's primary beam at $28.2 \mathrm{GHz}$, so this does not rule out the possibility that it shares the same structure at $z \sim 3.09$. We set a $3 \sigma$ upper limit of $I_{\mathrm{CO} 1-0}<0.41 \mathrm{Jy} \mathrm{km} \mathrm{s}^{-1}$ where we have adopted a line width of $800 \mathrm{~km} \mathrm{~s}^{-1}$, typical for SMGs (e.g. Greve et al. 2005).

SMMJ190827.5+721928, in the 6C 1909+72 field, is sufficiently bright that we would expect to detect its ${ }^{12} \mathrm{CO} J=1-0$ emission, given our $\Delta v \sim 20000 \mathrm{~km} \mathrm{~s}^{-1}(3.39<z<3.75)$ of velocity (redshift) coverage in this field, if it were to have a similar ratio of $S_{\mathrm{CO} 1-0} / S_{850 \mu \mathrm{m}}$ and be part of the same structure as the nearby $\mathrm{H}_{z} \mathrm{RG}$. We searched the ${ }^{12} \mathrm{CO} J=1-0$ velocity cube at the position of the SMG, at spectral resolutions ranging from 95 to $380 \mathrm{~km} \mathrm{~s}^{-1}$. Despite being amongst the brightest known SMGs, its position is ill-defined since it lacks a convincing counterpart in the available Spitzer 3.6- to $24-\mu \mathrm{m}$ imaging. The most convincing peak lies at $v_{\mathrm{obs}}=25.124 \pm 0.006 \mathrm{GHz}$, which would correspond to $I_{\mathrm{CO} 1-0}=$ $0.110 \pm 0.033 \mathrm{Jy} \mathrm{km} \mathrm{s}^{-1}$ at $z=3.588 \pm 0.001$ (after primary beam correction). The best-fitting line width $\left(430 \pm 120 \mathrm{~km} \mathrm{~s}^{-1}\right)$ would be narrower than the majority of $\mathrm{SMG}^{12} \mathrm{CO} J=3-2$ lines (Greve et al. 2005; Bothwell et al. 2012) and we do not view this as a robust detection, but it would be an obvious place to begin searching for ${ }^{12} \mathrm{CO} J=4-3$ with IRAM's new WideX correlator. Several other $2-3 \sigma$ peaks are also present, but none with a line width even remotely commensurate with an SMG (e.g. Greve et al. 2005). The $1 \sigma I_{\mathrm{CO}}$ level for a line of width $800 \mathrm{~km} \mathrm{~s}^{-1}$ is $0.06 \mathrm{Jy} \mathrm{km} \mathrm{s}^{-1}$. We are forced to conclude that the presence of two unusually bright SMGs in this field may be due to the chance alignment of distant starbursts.

The lack of a convincing detection of ${ }^{12} \mathrm{CO}$ towards the brightest SMG in the vicinity of 6C $1909+72$ casts doubt on the idea that it co-habits a proto-cluster environment with the radio galaxy (Stevens et al. 2003). However, our Herschel imaging does hint at the presence of a dust-rich strand of the cosmic web centred on $6 \mathrm{C} 1909+72$. Fig. 5 reveals a large $(\approx 500 \mathrm{kpc})$ red structure, the majority of which is co-aligned with a prominent extension $(\sim 150 \mathrm{kpc}$, somewhat larger than the $\sim 50-\mathrm{kpc}$ scale of the northern radio jet recall Fig. 1) seen previously at $850 \mu \mathrm{m}$ (Stevens et al. 2003). We might imagine this as a filament of gas that is being accreted slowly on to the AGN host galaxy, but in our view it is no less likely that this structure is due to the outflow of metal-rich material from the radio galaxy host, heated by a network of unresolved LIRGs. A dusty outflow could be driven by radiation pressure from a strong nuclear starburst and/or AGN on dust (e.g. Prochaska \& Hennawi 2009; Lehnert 

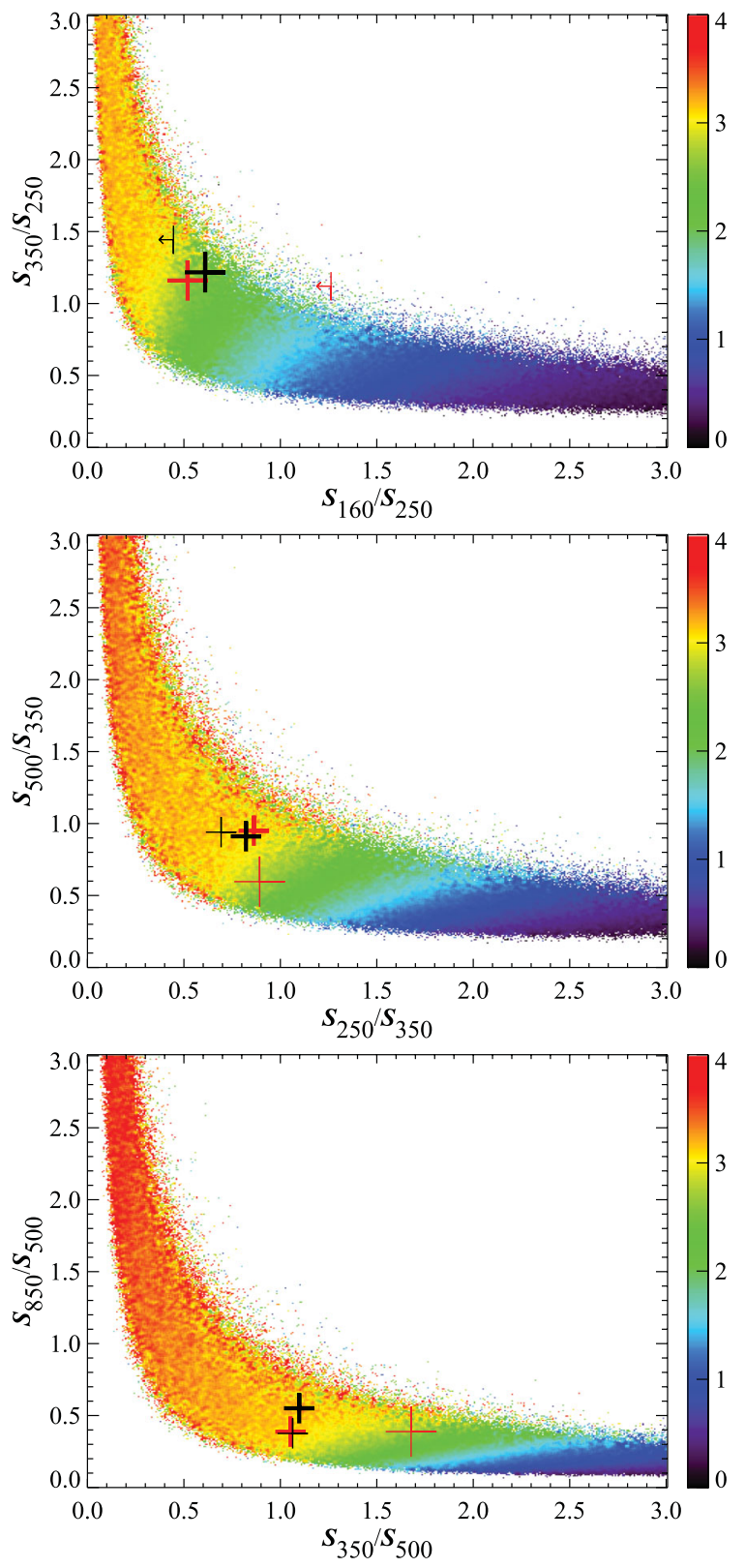

Figure 4. Colour-colour plots for the $\mathrm{H} z \mathrm{RGs}$ and their neighbouring SMGs, adapted from Amblard et al. (2010), together with a new plot designed to exploit our SCUBA $850-\mu \mathrm{m}$ photometry. For galaxies/bands without robust detections in Table 2, upper limits are shown (calculated as $S_{\lambda}+3 \sigma_{\lambda}$ ). The coloured backgrounds indicate the redshifts of model SEDs (Amblard et al. 2010). The red crosses represent B3 J2330+3927 (thick lines) and SMM J233019.1+392703, where the latter appears to lie at a lower redshift than the radio galaxy. The black crosses represent 6C 1909+72 (thick lines) and SMM J190827.5+721928, which have remarkably similar colours, consistent with a shared redshift of $\gtrsim 3$.

et al. 2011; Faucher-Giguere \& Quataert 2012; Roth et al. 2012; Wagner, Bicknell \& Umemura 2012) with material transported well beyond the AGN host galaxy, as witnessed in the near-IR on smaller scales for radio galaxies at $z \sim 2-3$ (Nesvadba et al. 2008; Harrison et al. 2012), for M 87 in the Virgo cluster (Simionescu et al. 2008, 2009; Werner et al. 2010), for the brightest cluster galaxy (BCG) in the Hydra A cluster (Kirkpatrick et al. 2009; Gitti et al. 2011) and for BCGs more generally (Kirkpatrick, McNamara \& Cavagnolo 2011; O'Sullivan et al. 2011). We note that the pres- ence of $\sim 3000 \mathrm{~km} \mathrm{~s}^{-1}$ absorption features ${ }^{5}$ against an unpolarized optical continuum led Dey (1997) to classify 6C 1909+72 as a broad-absorption-line radio galaxy, and is consistent with a powerful outflow. Of course, we must also bear in mind the well-rehearsed argument that radio galaxies are most easily discovered when their jets are encountering a working surface (e.g. Barthel \& Arnaud 1996).

\section{DISCUSSION}

\subsection{On the HzRG SEDs and $T_{\mathrm{b}}$ ratios}

Fig. 6 shows the observed radio-through-optical SEDs of 6 C 1909+72 and B3 J2330+3927. For both galaxies, the photometry between $3 \mu \mathrm{m}$ and $74 \mathrm{MHz}$ can be described adequately with a model comprising only synchrotron and thermal dust emission, following Kovács et al. (2010). Table 3 lists the results of a simultaneous fit to the following free parameters: the dominant cold dust temperature, $T_{\mathrm{d}}$, and the power-law index, $\gamma$, of a dust temperature distribution, $\mathrm{d} M_{\mathrm{d}} / \mathrm{d} T_{\mathrm{d}} \propto T_{\mathrm{d}}^{-\gamma}$, designed to offer a physically motivated treatment of the Wien side of the thermal emission spectrum (in Kovács et al., $T_{\mathrm{c}} \equiv T_{\mathrm{d}}$, the low-temperature cutoff of the distribution), the dust mass, $M_{\mathrm{d}}$ (for a characteristic photon crosssection to mass ratio, $\kappa_{850 \mu \mathrm{m}}=0.15 \mathrm{~m}^{2} \mathrm{~kg}^{-1}$, from Dunne, Eales \& Edmunds 2003, where the frequency dependence of the dust emissivity, $\beta$, was fixed to +1.5 ), and the synchrotron power-law index, $\alpha$. The resulting measurements of $L_{\mathrm{IR}}$ and $q_{\mathrm{IR}}$ (as defined by Helou, Soifer \& Rowan-Robinson 1985 , but where $S_{\text {IR }}$ is measured across $\lambda_{\text {rest }}=8-1000 \mu \mathrm{m}$, as is $\left.L_{\mathrm{IR}}\right)$ are also listed.

Both radio galaxies have warmer dust temperatures, $T_{\mathrm{d}}^{\mathrm{HzRG}} \sim$ $45 \mathrm{~K}$, than those seen for similarly luminous, dusty starbursts at $z$ $\sim 3\left(T_{\mathrm{d}}^{\mathrm{SMG}} \sim 35 \mathrm{~K}\right.$ when calculated using a power-law temperature distribution, as we have here - Magnelli et al. 2012). Determining the contribution of the AGN to $L_{\mathrm{IR}}$ is a long-standing problem that we cannot hope to solve without dramatically improved spatial resolution. The relatively warm dust does imply significant AGN contributions to $L_{\mathrm{IR}}$ for these two radio galaxies, though even this cannot be asserted with certainty since the brightest 100 - to $160-\mu \mathrm{m}$ emission in the B3 J2330+3927 system (Section 3.2) is centred near the gas-rich companion (which may contain its own AGN, if it is anything like the interacting system seen towards 4C 60.07-Ivison et al. 2008). On the other hand, the detection of dust and molecular gas towards the radio galaxy hosts makes it likely that significant levels of star formation are taking place in both. Given the strong dependence of $L_{\mathrm{IR}}$ on $T_{\mathrm{d}}$ (e.g. Eales et al. 2000), we might expect that the fraction of $L_{\mathrm{IR}}$ due to the AGN, $L_{\mathrm{IR}}^{\mathrm{AGN}} / L_{\mathrm{IR}} \approx 1-\eta\left(T_{\mathrm{d}}^{\mathrm{HzRG}} / T_{\mathrm{d}}^{\mathrm{SMG}}\right)^{-(4+\beta)}$, where $\eta \approx 0.5$ is the fractional AGN contribution to $L_{\mathrm{IR}}$ estimated for typical SMGs (e.g. Frayer et al. 1998). This suggests an $\approx 90$ per cent AGN-related contribution to $L_{\mathrm{IR}}$ for our $\mathrm{H} z \mathrm{RGs}$, which still leaves room for vigorous starburst activity in the radio galaxy hosts, with SFR $\sim 500 \mathrm{M}_{\odot} \mathrm{yr}^{-1}$ (Kennicutt 1998).

The superthermal $T_{\mathrm{b}}$ ratio, $r_{4-3 / 1-0}>2.7(3 \sigma)$, observed towards the AGN host galaxy, B3 J2330+3927, reveals the presence of highly excited molecular gas, given the excitation requirements of ${ }^{12} \mathrm{CO} J=4-3\left(E_{4-3} / k \sim 55 \mathrm{~K}\right.$ and $\left.n_{\text {crit }} \sim 1.9 \times 10^{4} \mathrm{~cm}^{-3}\right)$.

\footnotetext{
${ }^{5}$ For an $\sim 3000 \mathrm{~km} \mathrm{~s}^{-1}$ outflow, with a dynamical time-scale of a few $\times 10^{8} \mathrm{yr}$, a single epoch of starburst and/or AGN activity would be sufficient to generate a structure on the scale seen here.
} 

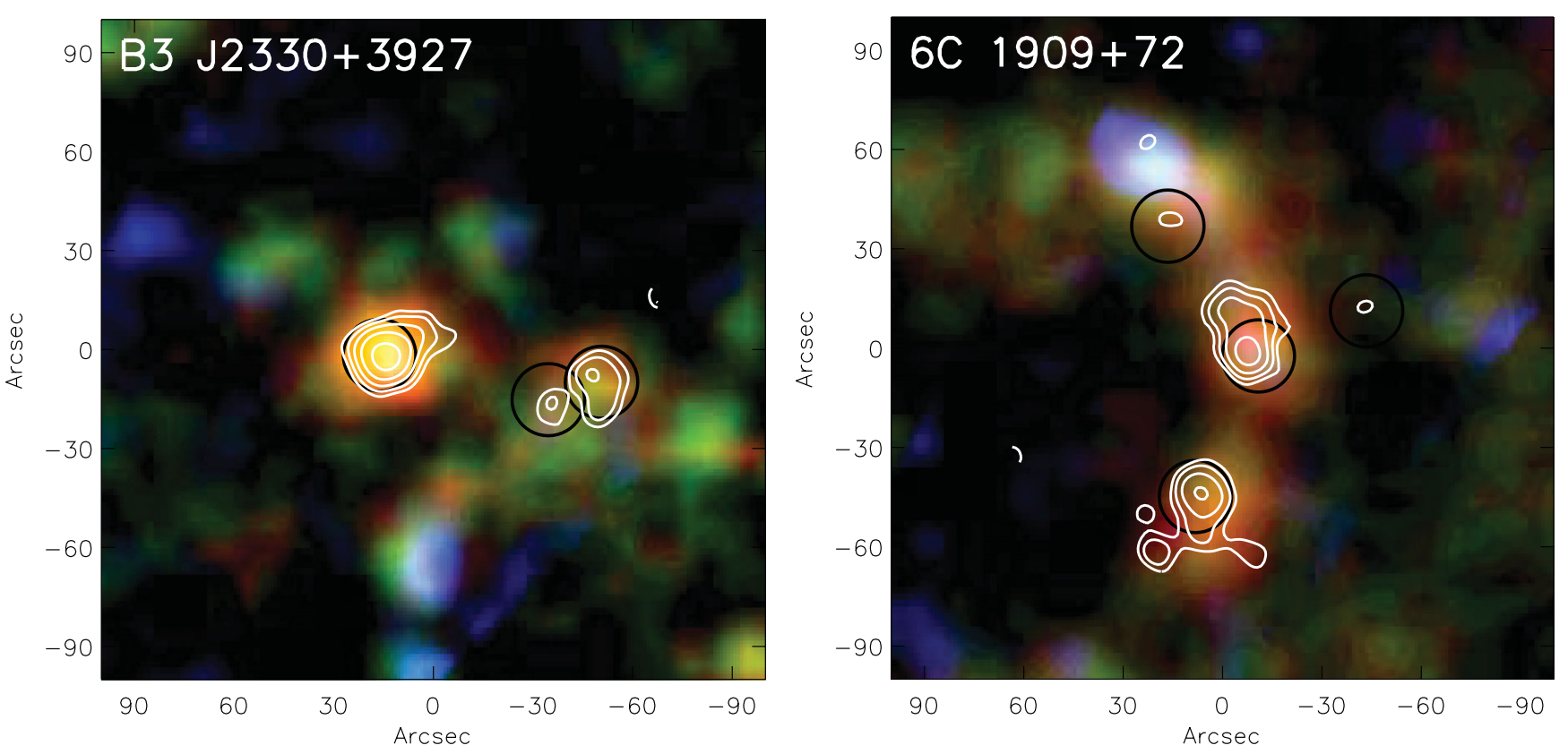

Figure 5. $850-\mu \mathrm{m}$ continuum emission ( $\sqrt{2}$-spaced contours, starting at $3 \sigma$; Stevens et al. 2003) for the fields surrounding B3 J2330+3927 (left) and 6C 1909+72 (right), superimposed on false-colour images made using the Herschel 160-, 250- and 350- $\mu \mathrm{m}$ imaging, described in Section 2.3, convolved to the 350- $\mu \mathrm{m}$ spatial resolution. N is up; E is left. The SMGs identified by Stevens et al. (2003) are circled. Unsurprisingly, these are revealed here as the reddest objects in each field. The red structure apparent in the 6C 1909+72 field is co-aligned with both the radio jets (recall Fig. 1) and a prominent extension seen previously at $850 \mu \mathrm{m}$. A less prominent extension is seen to the WNW of B3 J2330+3927.
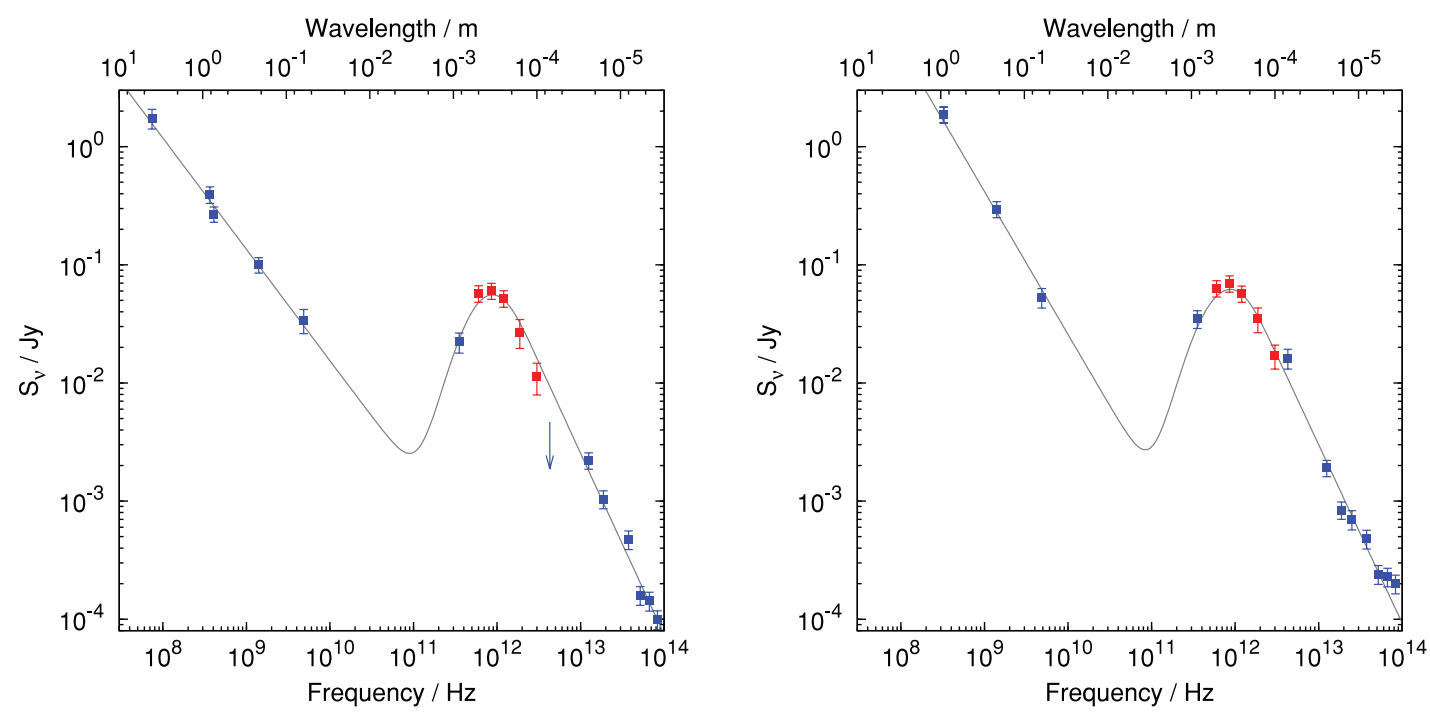

Figure 6. Radio-through-optical SEDs of the radio galaxies, B3 J2330+3927 (left) and 6C 1909+72 (right). Note that all quantities are observed rather than rest frame. Their entire 3- $\mu \mathrm{m}$ to 74-MHz SEDs can be fitted adequately (see Section 4.1) using only thermal dust and synchrotron emission components, whose characteristics are listed in Table 3. Our new measurements from PACS and SPIRE - which probe the SED peaks, constraining $L_{\mathrm{IR}}$ and $T_{\mathrm{d}}-$ are shown in red. Lacking the spatial resolution to account for contamination of flux densities for B3 J2330+3927 by component c (JVLA 233024.69+392708.6), we fit to the summed flux densities of both galaxies.

Even for $6 \mathrm{C} 1909+72$, the $T_{\mathrm{b}}$ ratio, $r_{4-3 / 1-0}=0.76 \pm 0.18$, is significantly higher than the average value seen for SMGs by Bothwell et al. (2012). IRAM PdBI and VLA observations of 4C 41.17 at $z=3.80$ revealed a similar story, with $r_{4-3 / 1-0}$ likely superthermal (Papadopoulos et al. 2005). In comparison, the same line ratio averaged over the entire Milky Way is $r_{4-3 / 1-0} \sim 0.1-0.2$ (Fixsen,
Bennett \& Mather 1999), indicative of quiescent gas in the interstellar medium (ISM).

In contrast to B3 $\mathrm{J} 2330+3927$, the $T_{\mathrm{b}}$ ratio that we find for its companion galaxy, JVLA $233024.69+392708.6\left(r_{4-3 / 1-0}=0.43 \pm\right.$ 0.11 , is consistent with the average value seen for SMGs and with star-forming regions within nearby starburst galaxies such 
Table 3. $T_{\mathrm{b}}$ ratios and parameters from SEDs.

\begin{tabular}{lcc}
\hline Parameter & 6C $1909+72$ & B3 J2330+3927 \\
\hline$r_{4-3 / 1-0}$ & $0.76 \pm 0.18$ & $3 \sigma>2.7$ \\
$\chi_{\text {red }}^{2}$ & 1.14 & 1.00 \\
$T_{\mathrm{d}}(\mathrm{K})$ & $45.7 \pm 1.3$ & $41.4 \pm 1.1$ \\
$\log M_{\mathrm{d}}\left(\mathrm{M}_{\odot}\right)$ & $9.37 \pm 0.04$ & $9.26 \pm 0.17$ \\
$\gamma$ & $5.51 \pm 0.05$ & $5.54 \pm 0.04$ \\
$\alpha$ & $-1.22 \pm 0.04$ & $-0.95 \pm 0.07$ \\
$q_{\mathrm{IR}}$ & $-1.02 \pm 0.04$ & $-0.40 \pm 0.11$ \\
$\log L_{\mathrm{IR}}\left(\mathrm{L}_{\odot}\right)$ & $13.69 \pm 0.08$ & $13.52 \pm 0.07$ \\
\hline
\end{tabular}

as M 82 and NGC 253, which have values in the range $\sim 0.5$ 0.8 (Güsten et al. 1993; Mao et al. 2000). The $T_{\mathrm{b}}$ ratio in JVLA $233024.69+392708.6$ is thus consistent with gas heated by UV radiation from star formation, i.e. less extreme ISM conditions than those of the radio galaxy.

$T_{\mathrm{b}}$ ratios as high as that observed towards B3 J2330+3927 are expected in environments where the gas energetics are dominated by X-rays. These can penetrate large columns of gas and maintain a high kinetic gas temperature, $T_{\mathrm{k}}$, much deeper into molecular clouds than far-UV (FUV) photons (e.g. Meijerink, Spaans \& Israel 2006; Schleicher, Spaans \& Klessen 2010; van der Werf et al. 2010), with this gas thermally decoupled from the cooler dust. Powerful radio-loud AGN are often found to have high X-ray luminosities, capable of powering extended $\mathrm{X}$-ray-dominated regions; while B3 J2330+3927 has no X-ray measurements, this is one possible explanation for the observed $r_{4-3 / 1-0}$.

Another possible explanation is shock-excited gas (e.g. Flower \& Pineau Des Forêts 2010) caused by its radio jet ramming into the molecular environment, bearing in mind that the mechanical power of a radio jet can be orders of magnitude larger than that of its synchrotron emission. An example of this is observed in the powerful radio galaxy, 3C 293, which has Milky Way-like levels of star formation and a very low X-ray luminosity (see also Matsushita et al. 2004, regarding the central region of M 51). Its superthermal ${ }^{12} \mathrm{CO}$ (as measured in ${ }^{12} \mathrm{CO} J=1-0$ and $J=4-3$ ) has been attributed to turbulent heating from the dissipation of shocks caused by the interaction of its powerful radio jet with its molecular gas (Papadopoulos et al. 2008; Nesvadba et al. 2010; Guillard et al. 2012) - an interaction that seemingly also drives an $\sim 1400 \mathrm{~km} \mathrm{~s}^{-1}$ outflow in $\mathrm{HI}$ (Morganti et al. 2003). Such mechanisms are often proposed to provide the feedback needed to reconcile models of galaxy formation with a variety of observations (e.g. Granato et al. 2004; McNamara $\&$ Nulsen 2012; Fabian 2012 and references therein).

A large fraction of the radio emission from B3 J2330+3927 comes from its core $(\sim 50$ per cent at $8.4 \mathrm{GHz})$ and it displays an unusually one-sided jet: 'one of the most asymmetric radio structures ever reported for a type II AGN' (Pérez-Torres \& De Breuck 2005). This may be due to relativistic Doppler beaming (e.g. Riley \& Warner 1990) or because of the launch mechanism (e.g. Chagelishvili, Bodo \& Trussoni 1996), but if a survey of such galaxies were to find relatively high $T_{\mathrm{b}}$ ratios relative to galaxies hosting two-sided jets, then it would suggest a more prosaic reason: a blockage that causes an unusually high fraction of the mechanical energy from AGN-driven outflow to be deposited into the molecular gas.

According to Wagner \& Bicknell (2011), the kinetic energy and momentum of a jet can be transferred to dense gas with a high efficiency (10-70 per cent), causing turbulent heating and shock excitation of the molecular gas and - in extreme cases - driving gas outflows and inhibiting star formation. The kinetic energy of the radio jet in B3 J2330+3927 can be estimated following Punsly (2005) and Punsly et al. (2008), assuming $S_{151 \mathrm{MHz}}=0.90 \mathrm{Jy}$ (from $S_{365 \mathrm{MHz}}=0.394 \mathrm{Jy}$ and $\alpha_{74 \mathrm{MHz}}^{365 \mathrm{MHz}}=-0.93$; Douglas et al. 1996; Cohen et al. 2007, though see also Bîrzan et al. 2008 and Cavagnolo et al. 2010). The jet's kinetic luminosity, $Q$, is then $\sim 2 \times$ $10^{46} \mathrm{erg} \mathrm{s}^{-1}$, roughly two orders of magnitude larger than the ${ }^{12} \mathrm{CO}$ $J=4-3$ luminosity, $L_{\mathrm{CO} 4-3}^{\prime}=3.4 \times 10^{44} \mathrm{erg} \mathrm{s}^{-1}$. This simplistic approach assumes that the 151-MHz emission comprises optically thin radiation from the lobes, whereas a substantial fraction may come from a Doppler-boosted jet. Nevertheless, over the $\sim 10-\mathrm{Myr}$ lifetime of the radio source we can expect a few $10^{60} \mathrm{erg}$ to be deposited into the multi-phase ISM (enough to quench a cooling flow for $\sim 10^{9} \mathrm{yr}$, as argued by McNamara et al. 2005). The turbulent kinetic energy of the molecular gas can be calculated through $E_{\text {kin }}^{\text {turb }}=\frac{3}{2} M_{\mathrm{H}_{2}} \sigma_{\mathrm{H}_{2}}{ }^{2}$, where $\sigma_{\mathrm{H}_{2}}=\mathrm{FWHM} / 2 \sqrt{2 \ln 2}$ is the velocity dispersion of the gas. From Section 3.2, $M_{\mathrm{H}_{2}}<2.3 \times 10^{10} \mathrm{M}_{\odot}$ so if $\sigma_{\mathrm{CO} 1-0} \sim \sigma_{\mathrm{CO} 4-3}=350 \mathrm{~km} \mathrm{~s}^{-1}$ and is solely due to the velocity dispersion of the gas (i.e. no components due to rotation or bulk outflow), then $E_{\text {kin }}^{\text {turb }}<10^{59} \mathrm{erg}$. We can thus conclude (as did Nesvadba et al. 2008, for an analogous situation) that the radio jet carries sufficient energy to explain the observed $\mathrm{CO}$ characteristics. The jet can influence the gas properties in precisely the way required to stop star formation, though questions clearly remain about the ultimate fate of the gas, and deeper observations are required to search for any high-velocity molecular component (see, e.g., Polletta et al. 2011).

Taking another approach, if we adopt a fiducial jet advance speed, $v_{\text {jet }} \sim 0.1 c$, then the gas is heated to $T_{\text {shock }} \approx 3 / 16 \mu m_{\mathrm{H}} / k v_{\text {jet }}^{2} \approx$ $10^{10} \mathrm{~K}$, where $\mu$ is the reduced mass and $k$ is Boltzmann's constant. For a large fraction of the gas that has been shock-heated by the jet, the density will be low $\left(10^{-2}-10^{-3} \mathrm{~cm}^{-3}\right.$; Wagner \& Bicknell 2011). This overpressurized gas will expand (Begelman \& Cioffi 1989), running into the denser, ambient ISM, which will be engulfed and destroyed by the resulting shock (e.g. Klein, McKee \& Colella 1994). The velocity of the shock that is driven into the dense clouds is $v_{\mathrm{s} \text {, cloud }}=v_{\text {wind }}\left(\rho_{\text {wind }} / \rho_{\text {cloud }}\right)^{1 / 2}$, where $\rho_{\text {wind }}$ is the density of the hot plasma created by the outward-moving radio jet and $\rho_{\text {cloud }}$ is the density of the engulfed ISM. For typical molecular clouds, $\rho_{\text {cloud }} \sim$ $10^{4} \mathrm{~cm}^{3}$, so we arrive at $v_{\mathrm{s} \text {, cloud }} \sim 20-40 \mathrm{~km} \mathrm{~s}^{-1}$ (Begelman \& Cioffi 1989). Such molecular shocks would be significant emitters in high$J$ CO lines (Flower \& Pineau Des Forêts 2010).

At what rate must the gas be shock-heated to maintain this $\mathrm{CO}$ emission? For a C-type shock wave with $v_{\mathrm{s} \text {, cloud }} \sim 40 \mathrm{~km} \mathrm{~s}^{-1}$ (assuming a lower velocity or a denser ambient ISM will make little difference, and a $J$-type shock will produce a line luminosity almost two orders of magnitude fainter), we find that to explain $L_{\mathrm{CO} 4-3}^{\prime}$ requires a mass shock rate of $\sim 30 \mathrm{M}_{\odot} \mathrm{yr}^{-1}$ (Flower \& Pineau Des Forêts 2010). Over the age of the jet, only a few $\times 10^{9} \mathrm{M}_{\odot}$ of gas needs to be shock heated, perhaps much less if this is a special, short-lived phase due to the initial passage of the radio jet. The gas may return to a higher density following the passage of the shock (Cooper et al. 2008), perhaps re-forming $\mathrm{H}_{2}$ (Guillard et al. 2009), so the amount of shock-heated gas required to explain the observed $T_{\mathrm{b}}$ ratios is not restrictive.

Whatever the excitation mechanism, the ${ }^{12} \mathrm{CO}$ optical depth must be moderate to low $\left(\tau_{1-0} \lesssim 1\right)$ since in the optically thick regime the $T_{\mathrm{b}}$ of a transition is equal to the excitation temperature, i.e. $T_{\mathrm{b}} \sim T_{\text {ex }}$ (with the latter being the same for all levels when thermalized - see Papadopoulos et al. 2011). In the optically thin case, 
$T_{\mathrm{b}}^{4-3} / T_{\mathrm{b}}^{1-0} \propto \tau_{4-3} / \tau_{1-0}$ and since $\tau_{\mathrm{J}+1, \mathrm{~J}} \propto(J+1)$ (at least up to $J \sim 7$ ), we find that $T_{\mathrm{b}}^{1-0}<T_{\mathrm{b}}^{4-3}$ (true for thermalized gas, but also for subthermal gas if the effect due to optical depth is sufficiently large). Optically thin ${ }^{12} \mathrm{CO}$ emission can be achieved if the medium is highly turbulent, as is the case for the Galactic Centre a local region where the ${ }^{12} \mathrm{CO}$ ladder is observed to be superthermal. The superthermal $T_{\mathrm{b}}$ ratio observed towards B3 J2330+3927 is thus likely an indication of mechanical energy deposited into the ISM from the radio jet and/or the ongoing starburst, inducing a degree of turbulence sufficient to make the ${ }^{12} \mathrm{CO}$ lines optically thin, allied with a high density ( $\left.\geq n_{\text {crit }}\right)$ and a relatively high kinetic temperature; moreover, this must happen globally, rather than in some small fraction of the gas, as seen in the nucleus of M 82, for example (Knapp et al. 1980; Weiß et al. 2001).

\subsection{The merger fraction amongst starbursting HzRGs}

The degree to which various populations of star-forming galaxies are driven by violent mergers of gas-rich galaxies or by the accretion of cold gas streams - sometimes smooth, sometimes clumpy - via dark matter filaments into spiral discs (Dekel, Sari \& Ceverino 2009) has been a topic of intense debate in recent years. Even for SMGs - the most extreme star formation events in the Universe, where the evidence for merger-driven activity was thought by many to be overwhelming (Engel et al. 2010) - the seeds of doubt have been sown.

What of radio galaxies? We cannot generalize to the entire population, even though that population is small in number: the timeconsuming interferometric observations of molecular gas that are capable of laying bare the processes leading to star formation have generally been obtained towards systems from which rest-frame FIR emission has already been detected, i.e. we are limited to systems pre-selected to be undergoing intense starbursts. Even so, it is interesting to add our findings to those studies of $z \gtrsim 2$ radio galaxies in the literature and explore the overall statistics. Take the eight systems listed in Section 1 in order of decreasing redshift.

(i) The interferometric ${ }^{12} \mathrm{CO} J=4-3$ study of $4 \mathrm{C} 41.17$, at $z=$ 3.80, by De Breuck et al. (2005) revealed activity driven by a merger between two massive, gas-rich galaxies separated by $\sim 2 \operatorname{arcsec}$ ( $\sim 13 \mathrm{kpc})$ and $\sim 400 \mathrm{~km} \mathrm{~s}^{-1}$.

(ii) High-resolution interferometric imaging of $4 \mathrm{C} 60.07$ at $z=$ 3.79 by Ivison et al. (2008) revealed two components of roughly equal integrated flux, separated by $\sim 30 \mathrm{kpc}$ - evidence of an earlystage merger between the host galaxy of an actively fuelled black hole (the $\mathrm{Hz} \mathrm{RG}$ ), and a gas-rich starburst/AGN, caught prior to its eventual equilibrium state. This separation is typical of SMGs with multiple radio identifications (Ivison et al. 2007) and for the intense burst of star formation near first passage in merger simulations (e.g. Springel, Di Matteo \& Hernquist 2005b).

(iii) Smith et al. (2010) use rest-frame UV spectroscopy to show that $6 \mathrm{C} 1909+72$, at $z=3.53$, comprises two galaxies separated by $\sim 1300 \mathrm{~km} \mathrm{~s}^{-1}$ along the line of sight, with the radio-loud AGN hosted by the more distant component.

(iv) TN J0121+1320 at $z=3.52$ was detected in ${ }^{12} \mathrm{CO} J=4-3$ by De Breuck et al. (2003b). There were no obvious signs of a gas-rich companion, but the available sensitivity, velocity coverage and spatial resolution were poor compared to recent studies.

(v) We have shown here that the immediate environment of B3 J2330+3927 at $z=3.09$ contains at least two gas-rich components, again separated by $\sim 30 \mathrm{kpc}$. (vi) Nesvadba et al. (2009) presented interferometric ${ }^{12} \mathrm{CO}$ imaging of the $z=2.58$ radio galaxy, TXS $0828+193$, finding two large gas reservoirs separated by $720 \mathrm{~km} \mathrm{~s}^{-1}$ along a shared line of sight roughly $80 \mathrm{kpc} \mathrm{SW}$ of the AGN host galaxy, which was itself detected only in synchrotron at $96.6 \mathrm{GHz}$.

(vii) 53W002 at $z=2.39$ was observed and detected in ${ }^{12} \mathrm{CO}$ (Yamada et al. 1995; Scoville et al. 1997) without knowledge of its FIR luminosity, though it was known to sit amongst a clump of Ly $\alpha$ emitting galaxies (Pascarelle et al. 1996) through which the radio galaxy is suspected to be assembling a large fraction of its eventual stellar mass through mergers (e.g. Motohara et al. 2001). At least one of these $-330 \mathrm{kpc}$ and $\gtrsim 300 \mathrm{~km} \mathrm{~s}^{-1}$ from $53 \mathrm{~W} 002$ - is FIRluminous (Smail et al. 2003). Based on their ${ }^{12} \mathrm{CO}$ imaging, Scoville et al. (1997) concluded 'it is clear that the emission is resolved', but this was not corroborated by Alloin et al. (2000). However, with an $\sim 6$-arcsec synthesized beam the latter data would be insensitive to close companions.

(viii) Finally, Hubble Space Telescope near-IR imaging of MRC 0152-209 at $z=1.92$ revealed a morphology consistent with an advanced-stage merger (Pentericci et al. 2001) where we would expect the gas to have been driven into a compact configuration, well within the 7- to 10-arcsec FWHM synthesized beam of the available ${ }^{12} \mathrm{CO}$ observations (Emonts et al. 2011).

Overall, we have six mergers or close galaxy pairs, one ambiguous case and one case where no merger activity can be discerned (albeit with relatively poor sensitivity and resolution available). The statistics suggest, then, that violent interactions are as ubiquitous amongst starbursting $\mathrm{H}_{z} \mathrm{RG}$ as they are amongst SMGs (Engel et al. 2010).

\section{CONCLUSIONS}

Combining our new interferometric ${ }^{12} \mathrm{CO}$ data for two distant radio galaxies with those for six more, reported elsewhere, we find that activity in starbursting radio-loud AGN at high redshift is driven by the merger or interaction of two or more systems in which significant masses of molecular gas and stars have already formed, rather than the steady ${ }^{6}$ accretion of cold gas from the cosmic web.

We introduce a new colour-colour diagnostic plot to constrain the redshifts of several distant, dusty galaxies found previously in our target fields. We conclude that the SMG south of 6C 1909+72 likely shares the same node or filament, but we fail to detect this FIR-luminous galaxy in ${ }^{12} \mathrm{CO}$, despite $\sim 20000 \mathrm{~km} \mathrm{~s}^{-1}$ of velocity coverage.

We introduce a new colour-colour diagnostic plot, exploiting the power of 350- to $850-\mu \mathrm{m}$ photometry to constrain the redshifts of several distant, dusty galaxies found previously in our target fields. We conclude that the bright SMG near 6C 1909+72 likely shares the same node or filament of the cosmic web at $z \sim 3.5$ as the signpost AGN. However, we fail to detect this SMG in ${ }^{12} \mathrm{CO} J=$ $1-0$, despite our $\sim 20000 \mathrm{~km} \mathrm{~s}^{-1}$ of velocity coverage.

Also in the 6C 1909+72 field, we find an unusually large, red dust feature, aligned with the radio jet. We suggest that metalrich material may have been dispersed on $\gtrsim 100-\mathrm{kpc}$ scales by a collimated outflow, reminiscent of the jet-oriented metal enrichment seen in X-ray observations of local cluster environments.

We find that ${ }^{12} \mathrm{CO}$ brightness temperature ratios in the host galaxies of radio-loud AGN are significantly higher than those seen in

\footnotetext{
${ }^{6}$ Often denoted 'secular', which in astronomy indicates that a process occurs continuously, as opposed to a discrete or periodic event such as a merger.
} 
similarly intense starbursts where AGN activity is less pronounced. In our most extreme example, the superthermal $T_{\mathrm{b}}$ ratio suggests that significant energy is being deposited rapidly into the molecular gas via X-rays and/or mechanical ('quasar-mode') feedback from the AGN, leading to a high degree of turbulence globally and a low optical depth in ${ }^{12} \mathrm{CO}$ - feedback that will lead to the cessation of star formation on a time-scale commensurate with that of the jet activity, $\lesssim 10$ Myr.

\section{ACKNOWLEDGMENTS}

We are grateful to Arjun Dey, Axel Weiss and Padelis Papadopoulos for sharing their wisdom regarding superthermal $T_{\mathrm{b}}$ ratios and unpublished optical spectroscopy. We appreciate the remarkable efforts of the NRAO staff that have significantly upgraded what many of us already regarded as the finest telescope ever built. In particular, we thank Frazer Owen and Gustaaf Van Moorsel for their invaluable help with the data used here. IRS acknowledges support from STFC and through a Leverhulme Senior Fellowship. NS is the recipient of an Australian Research Council Future Fellowship. TRG acknowledges support from STFC, as well as IDA and DARK. This work is based on observations carried out with the Karl Janksy Very Large Array. The NRAO is a facility of the NSF operated under cooperative agreement by Associated Universities, Inc. It is also based on observations carried out with the IRAM Plateau de Bure Interferometer. IRAM is supported by INSU/CNRS (France), MPG (Germany) and IGN (Spain).

\section{REFERENCES}

Alloin D., Barvainis R., Guilloteau S., 2000, ApJ, 528, L81

Amblard A. et al., 2010, A\&A, 518, L9

Archibald E. N., Dunlop J. S., Hughes D. H., Rawlings S., Eales S. A., Ivison R. J., 2001, MNRAS, 323, 417

Barthel P. D., Arnaud K. A., 1996, MNRAS, 283, L45

Begelman M. C., Cioffi D. F., 1989, ApJ, 345, L21

Best P. N., Longair M. S., Röttgering H. J. A., 1998, MNRAS, 295, 549

Biggs A. D., Ivison R. J., 2008, MNRAS, 385, 893

Bîrzan L., McNamara B. R., Nulsen P. E. J., Carilli C. L., Wise M. W., 2008, ApJ, 686, 859

Blain A. W., 1999, MNRAS, 309, 955

Blundell K. M., Rawlings S., 1999, Nat, 399, 330

Bothwell M. S. et al., 2012, MNRAS, submitted

Carilli C. L., Hodge J., Walter F., Riechers D., Daddi E., Dannerbauer H., Morrison G. E., 2011, ApJ, 739, L33

Cavagnolo K. W., McNamara B. R., Nulsen P. E. J., Carilli C. L., Jones C., Bîrzan L., 2010, ApJ, 720, 1066

Chagelishvili G. D., Bodo G., Trussoni E., 1996, A\&A, 306, 329

Chapman S. C., Blain A. W., Smail I., Ivison R. J., 2005, ApJ, 622, 772

Cohen A. S., Lane W. M., Cotton W. D., Kassim N. E., Lazio T. J. W., Perley R. A., Condon J. J., Erickson W. C., 2007, AJ, 134, 1245

Cooper J. L., Bicknell G. V., Sutherland R. S., Bland-Hawthorn J., 2008, ApJ, 674, 157

Davis M., Efstathiou G., Frenk C. S., White S. D. M., 1985, ApJ, 292, 371

De Breuck C. et al., 2003a, A\&A, 401, 911 (DB03)

De Breuck C., Neri R., Omont A., 2003b, New Astron. Rev., 47, 285

De Breuck C., Downes D., Neri R., van Breugel W., Reuland M., Omont A., Ivison R., 2005, A\&A, 430, L1

Dekel A., Sari R., Ceverino D., 2009, ApJ, 703, 785

Dey A., 1997, in Arav N., Shlosman I., Weymann R. J., eds, ASP Conf. Ser. Vol. 128, Mass Ejection from Active Galactic Nuclei. Astron. Soc. Pac., San Francisco, p. 35

Douglas J. N., Bash F. N., Bozyan F. A., Torrence G. W., Wolfe C., 1996, AJ, 111, 1945

Downes D., Solomon P. M., 1998, ApJ, 507, 615
Dunlop J. S., Hughes D. H., Rawlings S., Eales S. A., Ward M. J., 1994, Nat, 370,347

Dunne L., Eales S. A., Edmunds M. G., 2003, MNRAS, 341, 589

Eales S., Lilly S., Webb T., Dunne L., Gear W., Clements D., Yun M., 2000, AJ, 120, 2244

Eales S., Bertoldi F., Ivison R., Carilli C., Dunne L., Owen F., 2003, MNRAS, 344, 169

Eales S. et al., 2010, PASP, 122, 499

Emonts B. H. C. et al., 2011, ApJ, 734, L25

Engel H. et al., 2010, ApJ, 724, 233

Evans A. S., Sanders D. B., Mazzarella J. M., Solomon P. M., Downes D., Kramer C., Radford S. J. E., 1996, ApJ, 457, 658

Fabian A. C., 2012, preprint (arXiv:e-prints)

Faucher-Giguere C.-A., Quataert E., 2012, preprint (arXiv:e-prints)

Fixsen D. J., Bennett C. L., Mather J. C., 1999, ApJ, 526, 207

Flower D. R., Pineau Des Forêts G., 2010, MNRAS, 406, 1745

Frayer D. T., Ivison R. J., Scoville N. Z., Yun M., Evans A. S., Smail I., Blain A. W., Kneib J., 1998, ApJ, 506, L7

Gitti M., Nulsen P. E. J., David L. P., McNamara B. R., Wise M. W., 2011, ApJ, 732, 13

Granato G. L., De Zotti G., Silva L., Bressan A., Danese L., 2004, ApJ, 600, 580

Greve T. R., Ivison R. J., Papadopoulos P., 2004, A\&A, 419, 99

Greve T. R. et al., 2005, MNRAS, 359, 1165

Greve T. R., Stern D., Ivison R. J., De Breuck C., Kovács A., Bertoldi F., 2007, MNRAS, 382, 48

Greve T. R., Pope A., Scott D., Ivison R. J., Borys C., Conselice C. J., Bertoldi F., 2008, MNRAS, 389, 1489

Griffin M. J. et al., 2010, A\&A, 518, L3

Guillard P., Boulanger F., Pineau Des Forêts G., Appleton P. N., 2009, A\&A, 502,515

Guillard P. et al., 2012, ApJ, 747, 95

Güsten R., Serabyn E., Kasemann C., Schinckel A., Schneider G., Schulz A., Young K., 1993, ApJ, 402, 537

Harris A. I., Baker A. J., Zonak S. G., Sharon C. E., Genzel R., Rauch K., Watts G., Creager R., 2010, ApJ, 723, 1139

Harrison C. M. et al., 2012, preprint (arXiv:e-prints)

Hatch N. A., Overzier R. A., Röttgering H. J. A., Kurk J. D., Miley G. K., 2008, MNRAS, 383, 931

Hatch N. A. et al., 2011, MNRAS, 410, 1537

Helou G., Soifer B. T., Rowan-Robinson M., 1985, ApJ, 298, L7

Hippelein H., Meisenheimer K., 1993, Nat, 362, 224

Hodge J. A., Carilli C. C., Walter F., de Blok W. J. G., Riechers D., Daddi E., 2011, preprint (arXiv:e-prints)

Holland W. S. et al., 1999, MNRAS, 303, 659

Ibar E. et al., 2010, MNRAS, 409, 38

Ivison R. J., Papadopoulos P., Seaquist E. R., Eales S. A., 1996, MNRAS, 278,669

Ivison R. J., Dunlop J. S., Smail I., Dey A., Liu M. C., Graham J. R., 2000, ApJ, 542, 27

Ivison R. J. et al., 2007, MNRAS, 380, 199

Ivison R. J. et al., 2008, MNRAS, 390, 1117

Ivison R. J., Papadopoulos P. P., Smail I., Greve T. R., Thomson A. P., Xilouris E. M., Chapman S. C., 2011, MNRAS, 412, 1913

Kennicutt R. C., Jr, 1998, ARA\&A, 36, 189

Kirkpatrick C. C., Gitti M., Cavagnolo K. W., McNamara B. R., David L. P., Nulsen P. E. J., Wise M. W., 2009, ApJ, 707, L69

Kirkpatrick C. C., McNamara B. R., Cavagnolo K. W., 2011, ApJ, 731, L23

Klamer I. J., Ekers R. D., Sadler E. M., Weiss A., Hunstead R. W., De Breuck C., 2005, ApJ, 621, L1

Klein R. I., McKee C. F., Colella P., 1994, ApJ, 420, 213

Knapp G. R., Leighton R. B., Wannier P. G., Phillips T. G., Huggins P. J., 1980, ApJ, 240, 60

Kovács A. et al., 2010, ApJ, 717, 29

Lehnert M. D., Tasse C., Nesvadba N. P. H., Best P. N., van Driel W., 2011, A\&A, 532, L3

Magnelli B. et al., 2012, A\&A, 539, A155 
Mao R. Q., Henkel C., Schulz A., Zielinsky M., Mauersberger R., Störzer H., Wilson T. L., Gensheimer P., 2000, A\&A, 358, 433

Matsuda Y. et al., 2009, MNRAS, 400, L66

Matsushita S. et al., 2004, ApJ, 616, L55

McNamara B. R., Nulsen P. E. J., 2012, New Journal of Physics, 14, 055023

McNamara B. R., Nulsen P. E. J., Wise M. W., Rafferty D. A., Carilli C., Sarazin C. L., Blanton E. L., 2005, Nat, 433, 45

Meijerink R., Spaans M., Israel F. P., 2006, ApJ, 650, L103

Miley G. K. et al., 2004, Nat, 427, 47

Morganti R., Oosterloo T. A., Emonts B. H. C., van der Hulst J. M., Tadhunter C. N., 2003, ApJ, 593, L69

Morton D. C., Noreau L., 1994, ApJS, 95, 301

Motohara K. et al., 2001, PASJ, 53, 459

Nesvadba N. P. H., Lehnert M. D., De Breuck C., Gilbert A. M., van Breugel W., 2008, A\&A, 491, 407

Nesvadba N. P. H. et al., 2009, MNRAS, 395, L16

Nesvadba N. P. H. et al., 2010, A\&A, 521, A65

Nguyen H. T. et al., 2010, A\&A, 518, L5

O’Sullivan E., Giacintucci S., David L. P., Vrtilek J. M., Raychaudhury S., 2011, MNRAS, 411, 1833

Overzier R. A. et al., 2008, ApJ, 673, 143

Papadopoulos P. P., Ivison R. J., 2002, ApJ, 564, L9

Papadopoulos P. P., Röttgering H. J. A., van der Werf P. P., Guilloteau S., Omont A., van Breugel W. J. M., Tilanus R. P. J., 2000, ApJ, 528, 626 (P00)

Papadopoulos P. P., Greve T. R., Ivison R. J., De Breuck C., 2005, A\&A, 444,813

Papadopoulos P. P., Kovacs A., Evans A. S., Barthel P., 2008, A\&A, 491, 483

Papadopoulos P. P., van der Werf P., Xilouris E. M., Isaak K. G., Gao Y., Muehle S., 2011, preprint (arXiv:e-prints)

Pascarelle S. M., Windhorst R. A., Keel W. C., Odewahn S. C., 1996, Nat, 383, 45

Penner K. et al., 2011, MNRAS, 410, 2749

Pentericci L., Van Reeven W., Carilli C. L., Röttgering H. J. A., Miley G. K., 2000, A\&AS, 145, 121

Pentericci L., McCarthy P. J., Röttgering H. J. A., Miley G. K., van Breugel W. J. M., Fosbury R., 2001, ApJS, 135, 63

Pérez-Torres M.-A., De Breuck C., 2005, MNRAS, 363, L41

Perley R. A., Chandler C. J., Butler B. J., Wrobel J. M., 2011, ApJ, 739, L1

Pilbratt G. L. et al., 2010, A\&A, 518, L1

Poglitsch A. et al., 2010, A\&A, 518, L2

Polletta M., Nesvadba N. P. H., Neri R., Omont A., Berta S., Bergeron J., 2011, A\&A, 533, A20

Prochaska J. X., Hennawi J. F., 2009, ApJ, 690, 1558
Punsly B., 2005, ApJ, 623, L9

Punsly B., Clarke T. E., Tingay S., Gutiérrez C. M., Rasmussen J., Colbert E., 2008, ApJ, 687, 162

Reuland M., Röttgering H., van Breugel W., De Breuck C., 2004, MNRAS, 353,377

Riechers D. A. et al., 2011a, ApJ, 733, L11

Riechers D. A., Hodge J., Walter F., Carilli C. L., Bertoldi F., 2011b, ApJ, 739, L31

Riley J. M., Warner P. J., 1990, MNRAS, 246, 1P

Roth N., Kasen D., Hopkins P. F., Quataert E., 2012, preprint (arXiv:e-prints)

Röttgering H. J. A., Lacy M., Miley G. K., Chambers K. C., Saunders R., 1994, A\&AS, 108, 79

Schleicher D. R. G., Spaans M., Klessen R. S., 2010, A\&A, 513, A7

Scoville N. Z., Yun M. S., Windhorst R. A., Keel W. C., Armus L., 1997, ApJ, 485, L21

Seaquist E. R., Ivison R. J., Hall P. J., 1995, MNRAS, 276, 867

Seymour N. et al., 2007, ApJS, 171, 353

Seymour N. et al., 2012, ApJ, in press (arXiv:1206.5821)

Simionescu A., Werner N., Finoguenov A., Böhringer H., Brüggen M., 2008, A\&A, 482, 97

Simionescu A., Werner N., Böhringer H., Kaastra J. S., Finoguenov A., Brüggen M., Nulsen P. E. J., 2009, A\&A, 493, 409

Smail I., Ivison R. J., Gilbank D. G., Dunlop J. S., Keel W. C., Motohara K., Stevens J. A., 2003, ApJ, 583, 551

Smail I., Chapman S. C., Blain A. W., Ivison R. J., 2004, ApJ, 616, 71

Smith D. J. B., Simpson C., Swinbank A. M., Rawlings S., Jarvis M. J., 2010, MNRAS, 404, 1089

Smith A. J. et al., 2012, MNRAS, 419, 377

Springel V. et al., 2005a, Nat, 435, 629

Springel V., Di Matteo T., Hernquist L., 2005b, MNRAS, 361, 776

Stevens J. A. et al., 2003, Nat, 425, 264

Tacconi L. J. et al., 2008, ApJ, 680, 246

Tielens A. G. G. M., Miley G. K., Willis A. G., 1979, A\&AS, 35, 153

van der Werf P. P. et al., 2010, A\&A, 518, L42

van Ojik R. et al., 1997, A\&A, 321, 389

Venemans B. P. et al., 2007, A\&A, 461, 823

Wagner A. Y., Bicknell G. V., 2011, ApJ, 728, 29

Wagner A. Y., Bicknell G. V., Umemura M., 2012, preprint (arXiv:e-prints)

Weiß A., Neininger N., Hüttemeister S., Klein U., 2001, A\&A, 365, 571

Werner N. et al., 2010, MNRAS, 407, 2063

Yamada T., Ohta K., Tomita A., Takata T., 1995, AJ, 110, 1564

Younger J. D. et al., 2008, ApJ, 688, 59

This paper has been typeset from a $\mathrm{T}_{\mathrm{E}} \mathrm{X} / \mathrm{L} \mathrm{T} \mathrm{E} \mathrm{X}$ file prepared by the author. 